\title{
Long-Range Correlations and Natural Time Series Analyses from Acoustic Emission Signals
}

\author{
Leandro Ferreira Friedrich ${ }^{1}$, Édiblu Silva Cezar², Angélica Bordin Colpo², Boris Nahuel Rojo Tanzi², Mario Sob- \\ czyk $^{2}$, Giuseppe Lacidogna ${ }^{3, *}$, Gianni Niccolini ${ }^{3}$, Luis Eduardo Kosteski ${ }^{4}$ and Ignacio Iturrioz ${ }^{2}$
}

1 Department of Mechanical Engineering, Federal University of Pampa (UNIPAMPA), Alegrete/RS 97546-550, Brazil; leandrofriedrich@unipampa.edu.br (L.F.F.)

2 Department of Mechanical Engineering, Federal University of Rio Grande do Sul (UFRGS), Porto Alegre/RS 90046-902, Brazil; ediblucezar@gmail.com (E.S.C.); angelica.colpo@ufrgs.br (A.B.C.); boris.rojotanzi@ufrgs.br (B.N.R.T.); mario.sobczyk@ufrgs.br (M.S.); ignacio@mecanica.ufrgs.br (I.I.)

3 Department of Structural, Geotechnical and Building Engineering, Politecnico di Torino, 10129 Turin, Italy; gianni.niccolini@polito.it (G.N.)

4 Engineering Post Graduation Program, Federal University of Pampa (UNIPAMPA), Alegrete/RS 97546-550, Brazil; luiskosteski@unipampa.edu.br (L.E.K.)

* Correspondence: giuseppe.lacidogna@polito.it (G.L.)

\begin{abstract}
This work focuses on analyzing acoustic emission (AE) signals as a means to predict failure in structures. Two main approaches are considered: (i) long-range correlation analysis using both the Hurst $(H)$ and the Detrended Fluctuation Analysis (DFA) exponents, and (ii) natural time domain (NT) analysis. These methodologies are applied to the data collected from two application examples: a glass fiber reinforced polymeric plate and a spaghetti bridge model, where both structures were subjected to increasing loads until collapse. A traditional (AE) signal analysis is also performed to reference the study of the other methods. Results indicate that the proposed methods yield a reliable indication of failure in the studied structures.
\end{abstract}

Keywords: acoustic emission; long-range correlations; natural time analysis; heterogeneous materials

\section{Introduction}

Damage process in heterogeneous material remains an open research problem. Classical theoretical methods rely on treating the material as a continuum, where macro parameters are regularized to facilitate the application of differential analysis, such as using homogenization [1-3] or Plasticity-inspired damage theories [4-6]. However, this approach contradicts experimental evidence, which shows that the process is spasmodic and has evident discontinuity patterns. Thus, even if these methods yield satisfactory results in some instances, much rich information is also missed regarding the inherent discontinuities involved in heterogeneous-material structures. In contrast, other strategies focus directly on the information contained in the space-temporal distribution of discontinuities. Probably, the first one is the inspiriting Bundle Model proposed by Daniels, 1945 [7] and nowadays explored by Hansen \& Prhadhan, 2015 [8]. This approach essentially consists of a set of thin rods (a "bundle") with random resistance levels, subject to crescent loads. The evolution of the damage within the set is represented by the number of rods breaking with time, indicating the system's behavior as it approaches catastrophic failure. Other inherently discrete methods that include the possibility of failure include Meshfree Methods, Peridynamics, and the Discrete Element Method. For an excellent revision regarding these approaches, refer to Jenabidehkordi, 2019 [9].

Acoustic Emission (AE) techniques have been developed since the 1940s to monitor damage processes in structures, especially those built from heterogeneous material [10]. It consists in detecting the elastic waves produced by the abrupt changes inside the 
material (rupture, dislocation, among other causes) using sensors on the surface's structure. Each "waves' source" is called an event. The register of an event in an AE sensor is a signal or hit. Notice that there is no guarantee that an isolated signal informs of only one event since the waves from different events can affect the sensor simultaneously. Damage evolution is represented by the spatial-temporal distribution of the events, identified by a series of global parameters calculated from these signals, the most popular one being called $b$-value. This parameter could be related to the fractal dimension where the damage develops [11], being regarded in several works as a reliable precursor for the collapse, such as in Carpinteri et al., 2009 [12]. Other parameters are also used with the same goal. See, for instance, Alava and Zapperi, 2006 [18] and Xu et al., 2021 [19].

There is an identifiable pattern in complex systems in several science fields as they come close to instability, as illustrated by Wilson, 1979 [20] with the normalization group theory. This method's central idea is that when a system approaches its critical collapse, its associated process has characteristics that do not depend on its geometry and boundary conditions because all scales are activated. This fact is observed in economics [21; 22], solid-state physics [23], and mechanical systems [24], among other fields.

Several AE parameters present typical behavior that indicates when a system is close to its critical state. In this work, we investigate the behavior of long-range correlations of the temporal series studied using Rescaled Range (R/S) analysis (or Hurst method - $H$ ) [25] and Detrended Fluctuation Analysis (DFA) [26]. In addition, we analyze the temporal series using the Natural Time (NT) domain, a tool introduced by Varostsos et al., 2001 [27] and subsequently employed by Potirakis et al., 2013 [28].

These two approaches are used to analyze AE signals in two examples from previous works, which employed classical AE techniques. First is a polymer plate reinforced by glass fibers studied by Friedrich et al., 2020 [29]. The second was previously analyzed by Tanzi et al. 2021 [30] and consists of a spaghetti bridge structure built from spaghetti sticks and monitored while the structure was loaded up to reach the failure. The proposed parameters from AE signals are of interest not only because of their potential as precursors of failure in the studied systems but also because they illustrate their underlying principle: all systems present the same basic patterns when they approach criticality. As shown in this work, this feature is an effective alternative to identify damage in structures.

\section{Theoretical background}

This section presents a brief description of the global AE parameters discussed here. The first one is the $b$-value, a classic approach that serves as a reference for the analyses provided through the proposed methods. Two of the latter are long-range correlation measures: the Detrended Fluctuation Analysis (DFA) and the Rescaled Range (R/S) or Hurst Analysis. The third one is the Natural Time approach.

\section{1. b-value analysis}

AE-data analysis can be based on several criteria: signal amplitude, duration, rise time, acoustic emission energy, and number of AE signals. One of the most used is the bvalue, which is calculated from AE amplitude distribution data by the power-law relationship between the number of events $(N)$ that exceeds a given amplitude $(A)$ and the amplitude of these events [31]. This relationship is commonly known as Gutenberg-Richter [32]:

$$
N(\geq A) \propto A^{-b}
$$

Figure 1 illustrates how the $b$-value varies during a typical damage process. According to Aki [11] and Carpinteri et al. [12, 33], its physical meaning is related to the fractal dimension $(D)$ of the domain from which the cracks emanate through the expression $D=2 b$. 


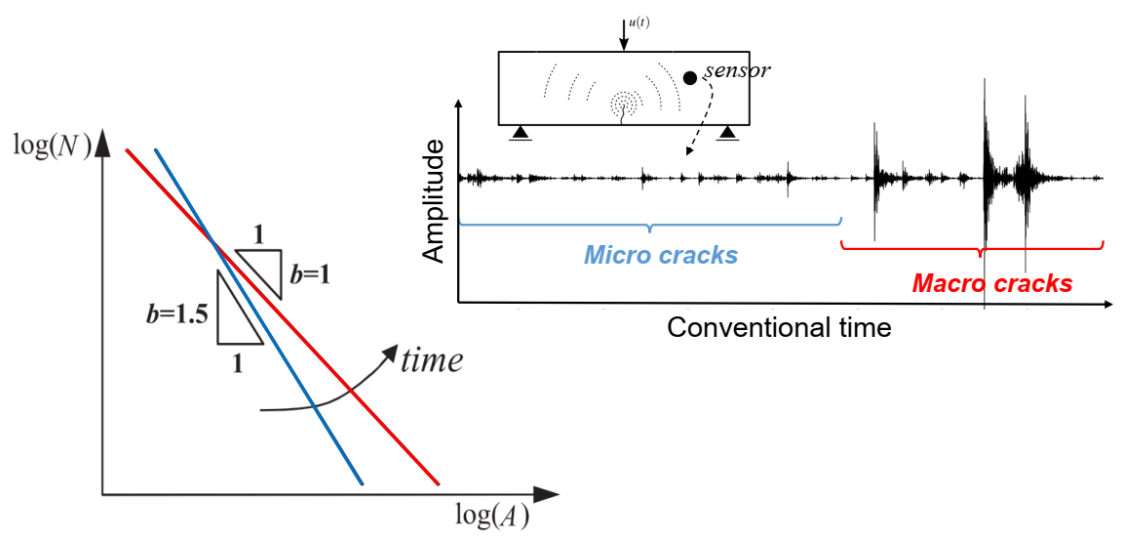

Figure 1. The $b$-value evolution and its physical meaning with respect to an acoustic emission signal.

At the beginning of the damage process, high $b$-values point out the occurrence of many small-amplitude AE events caused by micro-crack formation distributed throughout the whole structure's volume, implying $D=3$ and $D=1.5$. As the damage process evolves to produce the localization effect, i.e., events tend to emanate from a preferential region and form a "cloud" of micro-fissures [29]. Thus, macro-crack nucleation occurs, and the acoustic events tend to originate from a definite surface, i. e., $D=2$ and $D=1$. In terms of Equation (1), this is interpreted as an increment of high-amplitude events. The $b$ value is a classic parameter in structures monitoring, and therefore, it is used here as a reference for evaluating the proposed indexes' usefulness.

\subsection{Long-range correlations measures (DFA and Hurst)}

Complex systems appear in many sciences, in topics as varied as heart rate dynamics, DNA, neuron spiking, meteorology, human motor activity, seismic signals, and economic time series [34;35]. These systems are characterized by distinct effects such as nonlinearity, spontaneous order, and adaptation, among others, and can be studied through fractal properties [36; 37]. In AE signals like those in Figure 2, interpreting events as irregularities in a time series, their occurrence can be identified through the series properties, such as self-similarity and scale-invariance. For instance, long-range power-law correlations are present if a time series exhibits scale-invariant features [44;38]. The strength or persistence of such long-range correlations helps unravel or explain data behavior [38]. There are several methods to assess and quantify the strength of these correlations. This work uses two: Detrended Fluctuation Analysis (DFA) and rescaled range analysis (R/S).

The DFA is a method initially proposed by Peng et al., 1994 [26] to detect long-range correlations in time series with non-stationaries [37; 39-42]. Briefly, DFA fits a power law for estimating $\alpha$, which is a measure of long-range correlations. It is calculated by plotting in log-log axes the average fluctuations in the series, named FDFA, over different sampling scales (window sizes): $\alpha$ corresponds to the angular coefficient in the linear fit of FDFA versus windows size. Figure 2 exemplifies the application of the DFA method for a time series in the form $\{x(i)\}_{i=1}^{N_{\text {max }}}$, where $N_{\max }$ is the total number of points in the series, in the case of a signal series $x(i)$ with $N_{\max }=1000$, Figure 2a. The general steps for applying the DFA are as follows $[26 ; 37 ; 43]$ :

1. Integrate the series $x(i)$, obtaining $y(k)=\sum_{i=1}^{k} x(i)-\langle x\rangle$, where $k=1, \ldots, N_{\max }$ and $\langle x\rangle$ stands for the average of $x$, see Figure $2 b$.

2. Divide $\{y(k)\}_{i=1}^{N_{\max }}$ into non-overlapping windows of equal length $n$.

3. For each $n$-size window, fit a least-squares line to the integrated signal $y(k)$ (red line in Figure $2 \mathrm{~b})$. The sequence of fitted lines constitutes the trend series $y_{n}(k)$. 
Figure $2 \mathrm{~b}$ shows the integrated series with three different window sizes, $n=250, n=$ $100, n=50$.

4. Calculate the average fluctuation FDFA of the integrated series $y(k)$ around the trend series $y_{n}(k)$. Explicitly, $F_{D F A}(n)=\sqrt{\left(1 / N_{\max }\right) \sum_{k=1}^{N_{\max }}\left[y(k)-y_{n}(k)\right]^{2}}$.

5. Repeat step 4 for a broad range of scales (sizes of $n$ ) to provide a relationship between FDFA and $n$, i.e., $\operatorname{FDFA}_{\mathrm{DF}}(n)$.

6. Plot $\log \operatorname{FDFA}_{\mathrm{DF}}(n)$ versus $\log (n)$ (see Figure $2 \mathrm{c}$ ). If there is an obvious linear relationship between them, the slope of its least-squares fit estimates the scaling exponent $\alpha$.
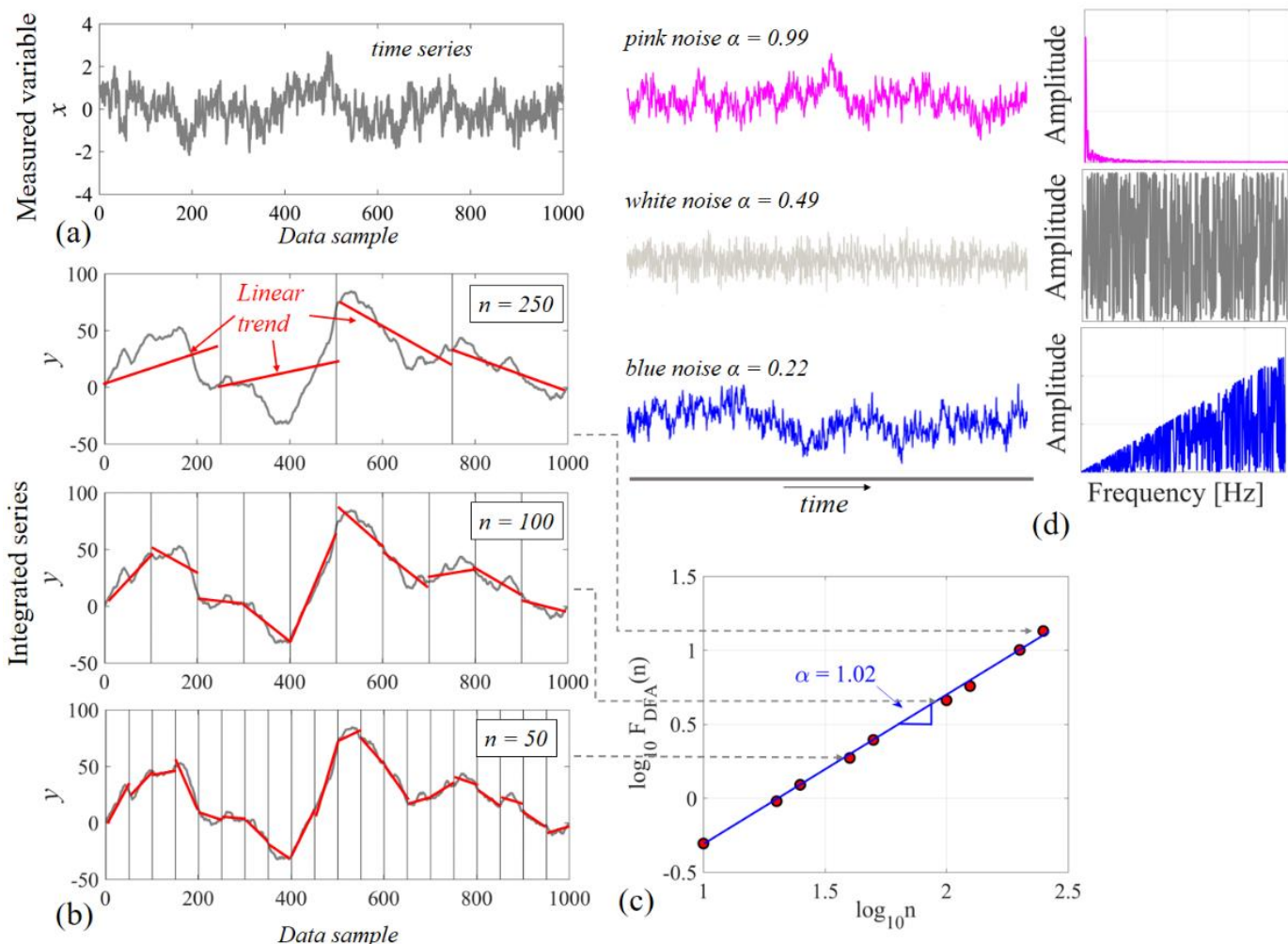

Figure 2. Graphical illustration of DFA steps. (a) Example of time series; (b) integrated series divided into different size windows with their corresponding linear-fit trends; (c) log-log plot of average fluctuations FDFA $(n)$ versus window sizes $n$. The estimated scaling exponent $\alpha$ is the slope of the least-squares fitted line; (d) representative examples of white (random), pink (fractal, long-range correlated, slightly persistent), and blue (anti- persistent) time and power-spectrum series. (Adapted from [44]).

As explained in detail in $[37 ; 44]$, applying the DFA methodology yields both a measure of persistence and an index of self-similarity in the time series. In short, $\alpha \approx 0.5$ indicates that the signal is uncorrelated or non-similar in variability structure, as in the case of white noise (Figure 3d). If the signal's variability structures are long-range correlated, persistent, or self-similar, i.e., if a small-amplitude signal sample is very likely to be followed by an even smaller one (or similarly, if a large-amplitude sample tends to be followed by an even larger one), one finds $\alpha>0.5$. That is the case with pink noise. Finally, if $\alpha<0.5$, signal correlation is anti-persistent (the blue noise in Figure 3d): a small-amplitude sample is more likely followed by a large-amplitude one, and vice-versa.

The so-called R/S analysis is also a classic approach to evaluate the persistence or selfsimilarity of a signal. This methodology was introduced by Hurst [25], an English hydrologist, in an extensive series of empirical studies involving the flow of the Nile River. Its basic idea is to determine how the range of the cumulative fluctuations depends on the length of the data subset under analysis [45], being illustrated in Figure 3 for the same time series discussed in Figure 2a, considering windows with size $n=250$ [46]. As in the 
DFA method, a time series of length $N_{\max }$ is divided into equal-length windows with size $n$. Next, for each window:

1. Take the mean $\langle x\rangle_{n}=1 / n \sum_{j=1}^{n} x(j)$ of the $n$-th window, marked as the solid red lines in Figure $3 b$.

2. Sum the differences from the mean to get the cumulative total $X(i, n)$ at each data point, from the beginning of the period to any desired point, i.e., $X(i, n)=\sum_{i=1}^{n} x(i)-\langle x\rangle_{n}$, Figure $3 b$.

3. Calculate the local range $R(n)=\max X(i, n)-\min X(i, n)$ as the maximum fluctuation of the sum of the deviation from the mean, where $\max X(i, n)$ and $\min X(i, n)$ are the maximum and minimum values of $X(i, n)$, respectively, and $1 \leq i \leq n$ (Figure 3b).

4. Take the standard deviation $\mathrm{S}(n)=\sqrt{1 / n \sum_{i=1}^{n}\left[x(i)-\langle x\rangle_{n}\right]^{2}}$ over the window to normalize the range relative to the input fluctuations in the series (dashed red lines in Figure 3b).

5. Rescale the range, that is, calculate $R(n) / \mathrm{S}(n)$.

6. Finally, calculate the mean value of the rescaled range for all windows, $n_{\mathrm{w}}$ (four in the case of Figure 3b):

$$
(R / \mathrm{S})_{n}=\frac{1}{n_{w}} \sum_{m=1}^{n_{w}} R_{m} / \mathrm{S}_{m}
$$

For windows with different lengths, the new dimensionless value R/S is well described by the following empirical relation, as illustrated in Figure 3c:

$$
R / \mathrm{S}_{n} \sim n^{H}
$$

In log-log axes, the slope of a least-squares regression is the estimate of the Hurst exponent $H$.

The parameter $H$ varies from 0 to 1 and has the same meaning as the $\alpha$ parameter (Figure $3 \mathrm{~d}$ ): $H \approx 0.5$ for the case of white noise, i.e., uncorrelated; $H>0.5$ if the process is persistent; and for an anti-persistent case, $H<0.5$. Moreover, it also expresses the time series 'fractal dimension $(d)$ from the relation $d=2-H$, where $1<d<2$ [47]. Thus, $d \rightarrow 1$ for persistent correlations, i.e., the series tends to a line, whereas it tends to a plane for anti-persistent correlations $(d \rightarrow 2)$.

About the two methodologies described above, $\alpha$ and $H$, some considerations are in order:

- Although this work is focused only on first-order fitting functions, as suggested in [43], higher-order fitting curves can also be used.

- Window sizes must be limited to avoid significant linearity variations in $(\operatorname{FDFA}(n)-n)$ and $(\mathrm{R} / \mathrm{S}-n)$. According to [48] and [49], the windows used here vary from $n_{\min }=10$ for the smallest to $n_{\max }=N_{\max } / 4$ for the largest. 

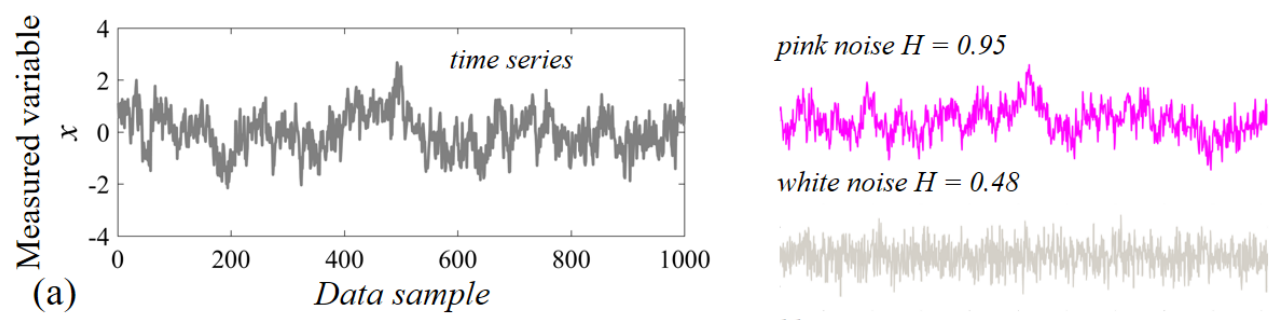

For $n=250$
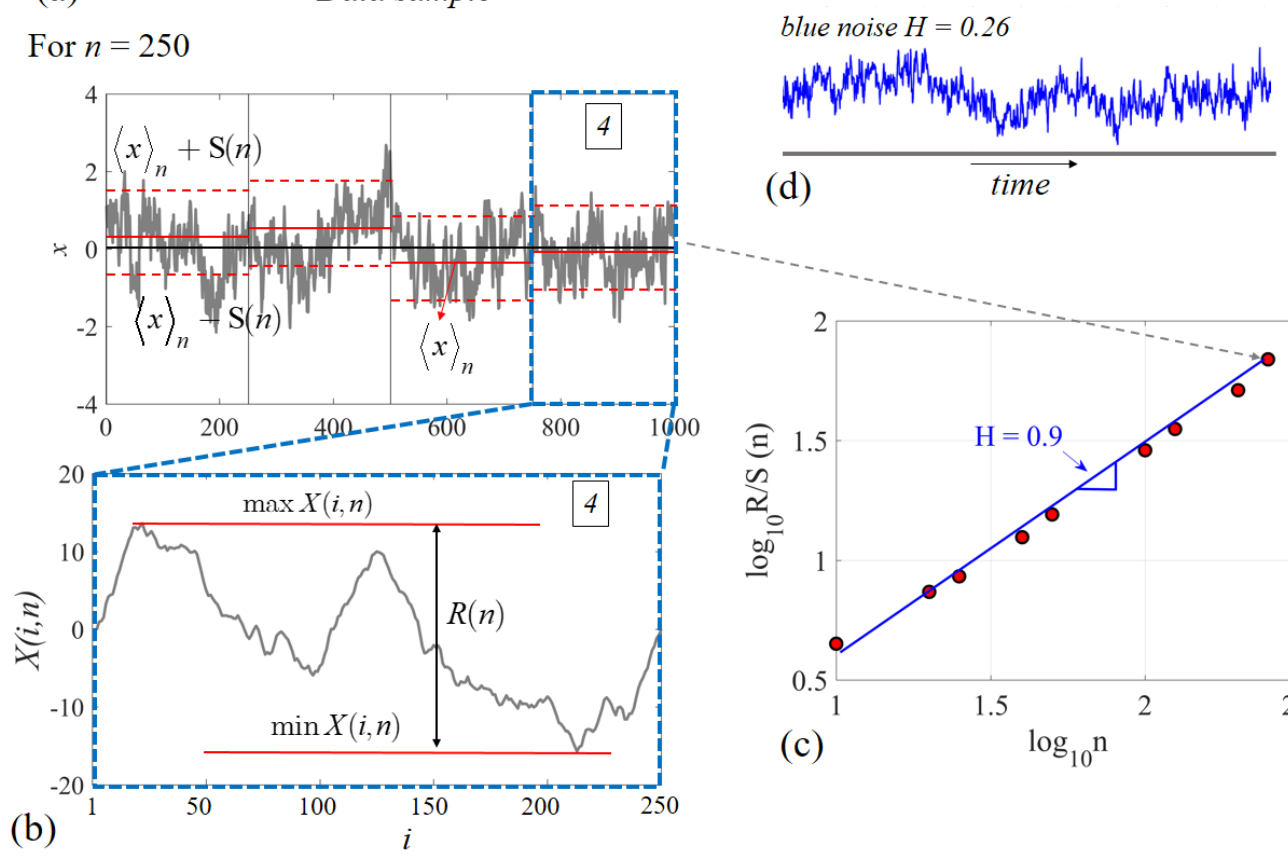

(d)

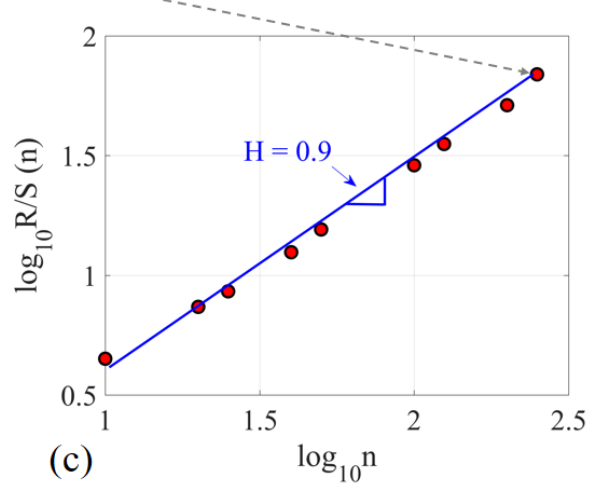

Figure 3. Basic parameters involved in standard R/S analysis: (a) a non-stationary time series $x(i)$; (b) Example of Hurst exponent considering $n=250$, where $\langle x\rangle$ is the mean and $\mathrm{S}(n)$ the standard deviation, $X(i, n)$ are the local differences accumulated from the mean, and $R(n)$ is the local range, that is, the difference between maximum and minimum $X(i, n)$ (adapted from [45]); (c) log-log plot of the rescaled range R/S versus window size $n$. The estimated scaling exponent $H$ is the slope of the least-squares fitting line; (d) representative examples of $H$ value for a white (random), pink (fractal, long-range correlated, slightly persistent), and blue (anti- persistent) time series.

\subsection{Natural-time analysis}

The analysis in the natural time domain allows identifying when a complex system enters a critical state [50]. For a time series comprising $N$ events, the natural time $\chi_{k}$ of the $k$-th event is defined as this event normalized, divided by the total number of the considered events, $\chi_{k}=k / N \quad[2]$, see Figure 4 . On the other hand, natural time $\chi_{k}$ serves as an index for the occurrence of the $k$-th event, Figure 4 . It is, therefore, smaller than, or equal to, unity $(0,1)[47 ; 51]$. We denote by $Q_{k}$ a quantity proportional to the energy of the individual $k$-th event, so its normalized energy is given by $p_{k}=Q_{k} / \sum_{n=1}^{N} Q_{n}$. In natural time analysis, the evolution of the pair $\left(\chi_{k}, p_{k}\right)$ is considered [47], see Figure 4.

The natural time $\chi_{k}$ 's variance $\kappa_{1}$ is given by:

$$
\kappa_{1}=\left\langle\chi^{2}\right\rangle-\langle\chi\rangle^{2} \equiv \sum_{k=1}^{N} p_{k}\left(\frac{k}{N}\right)^{2}-\left(\sum_{k=1}^{N} \frac{k}{N} p_{k}\right)^{2}
$$


$\kappa_{1}$ varies when a new $k$-th event occurs, once natural time $\chi_{k}$ changes from $k / N$ to $k /(N+1)$ and $p_{k}$ changes to $Q_{k} / \sum_{i=1}^{N} Q_{i}$. The called order parameter $\kappa_{1}$ may identify the approach of the dynamical system to a critical point [51].

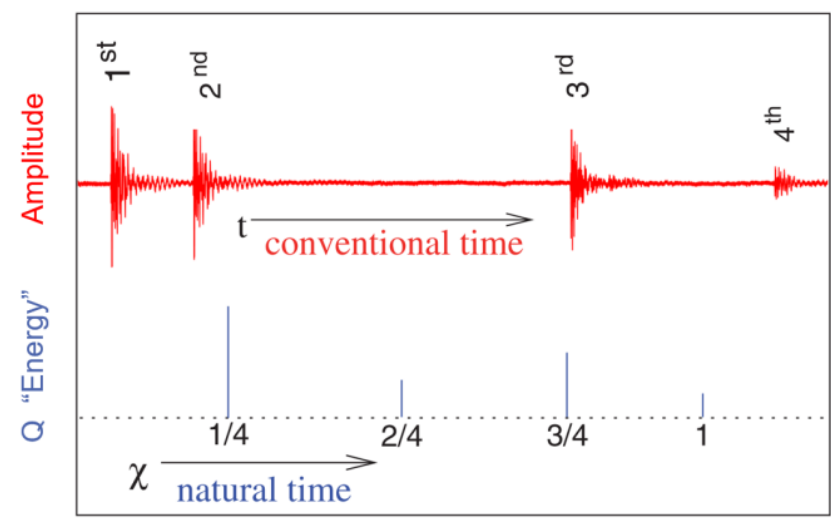

Figure 4. Natural time in the context of acoustic emission time series (adapted from [51]).

Moreover, the entropy $(S)$ (note that the letter $S$ is in italics, different from the standard deviation $S$ presented in the previous section) in natural-time domain is defined as,

$$
S=\langle\chi \ln \chi\rangle-\langle\chi\rangle \ln \langle\chi\rangle \equiv \sum_{k=1}^{N} p_{k} \chi_{k} \ln \chi_{k}-\left(\sum_{k=1}^{N} p_{k} \chi_{k}\right) \ln \left(\sum_{k=1}^{N} p_{k} \chi_{k}\right)
$$

Notice that the entropy in natural time refers to numerical signal data and does not directly relate to the property from traditional thermodynamics [52], being related to the information theory instead [53]. Like the variance $\kappa_{1}$, the entropy $S$ is also a dynamic parameter depending on the sequential order of events [54].

The third variance term considered in this method is the time-reversal entropy $S_{r e v}$. It is obtained by reversing the order of the energy data $\left(Q_{k}\right)$, calculating $S$ with this new series, and then subtracting the entropy from the original series from the result. According to Helman et al., 2020 [55], this entropy parameter is the most sensitive one to the ordering of the signal data.

It is possible to evaluate another order parameter, which describes the "average" distance $\langle D\rangle$ between the curves of the normalized power spectrum of the events [51],

$$
\Pi(\omega)=\left|\sum_{k=1}^{N} p_{k} e^{i \omega \frac{k}{N}}\right|^{2}
$$

where $\omega=2 \pi \phi \quad(\phi \in(0,0.5)$ standing for the frequency in natural time) and the ideal normalized power spectrum,

$$
\Pi(\omega)_{\text {ideal }}=\frac{18}{5 \omega^{2}}-\frac{6 \cos \omega}{5 \omega^{2}}-\frac{12 \sin \omega}{5 \omega^{3}}
$$

if $\omega \rightarrow 0$, Equation $(7)$ simplifies to $\Pi(\omega)_{\text {ideal }} \approx 1-0.07 \omega^{2}$. When $\Pi(\omega)$ approaches $\Pi(\omega)_{\text {ideal }}$ from below, the critical state is indicated.

Application of the natural time method to analyze AE signals can be described with aid from Figure 5. First, the events/hits are separated from the overall signal, Figure 5a. Then, they are ordered in the natural time domain, that is, in terms of $\chi_{k}$ and $Q_{k}$ (Figure $5 b)$. Next, of the order parameters, $\kappa_{1}$ and $\langle D\rangle$, and the entropy measurements $S$ and 
$S_{r e v}$ are calculated with Equations (4)-(7). Finally, according to Varotsos [56-59], the dynamical system is defined as in a true critical state ("tcrit" is achieved, see Figure $5 c$ ) if:

1. The "average" distance $\langle D\rangle$ becomes smaller than $10^{-2}$.

2. The variance $\kappa_{1}$, when descending from above, approaches 0.070 ;

3. The entropies $S$ and $S_{r e v}$ are both smaller than the entropy of uniform noise $S_{u}=(\ln 2 / 2)-1 / 4 \approx 0.0966$.

Figure 5 c shows an example of true coincidence, indicated as $t_{\text {crit, }}$ where all the criteria listed above are met. Furthermore, in Figure 5d, a typical sequence of the normalized power spectrum is presented, where it is expected that as it approaches the critical stage, the obtained data (dashed lines) reach the analytical solution (solid lines). It has been observed that the instant of (true) coincidence may also be invariant with the chosen threshold value [60]. This possibility will also be explored in the applications described in Section 3.

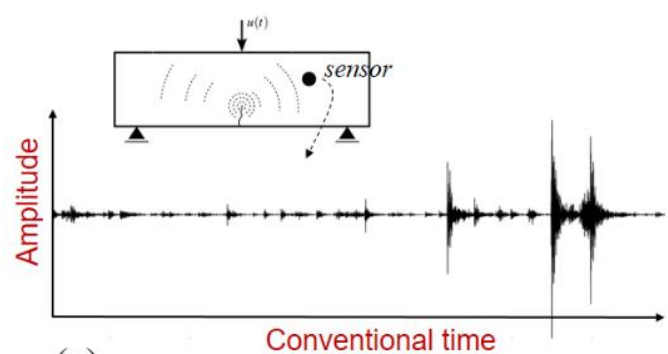

(a)

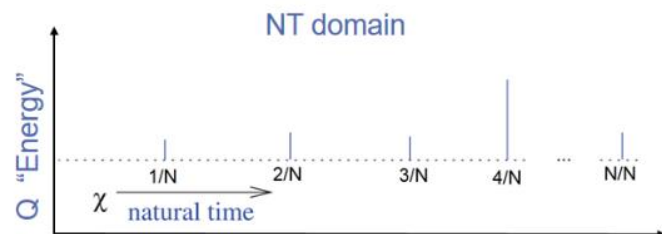

(b)
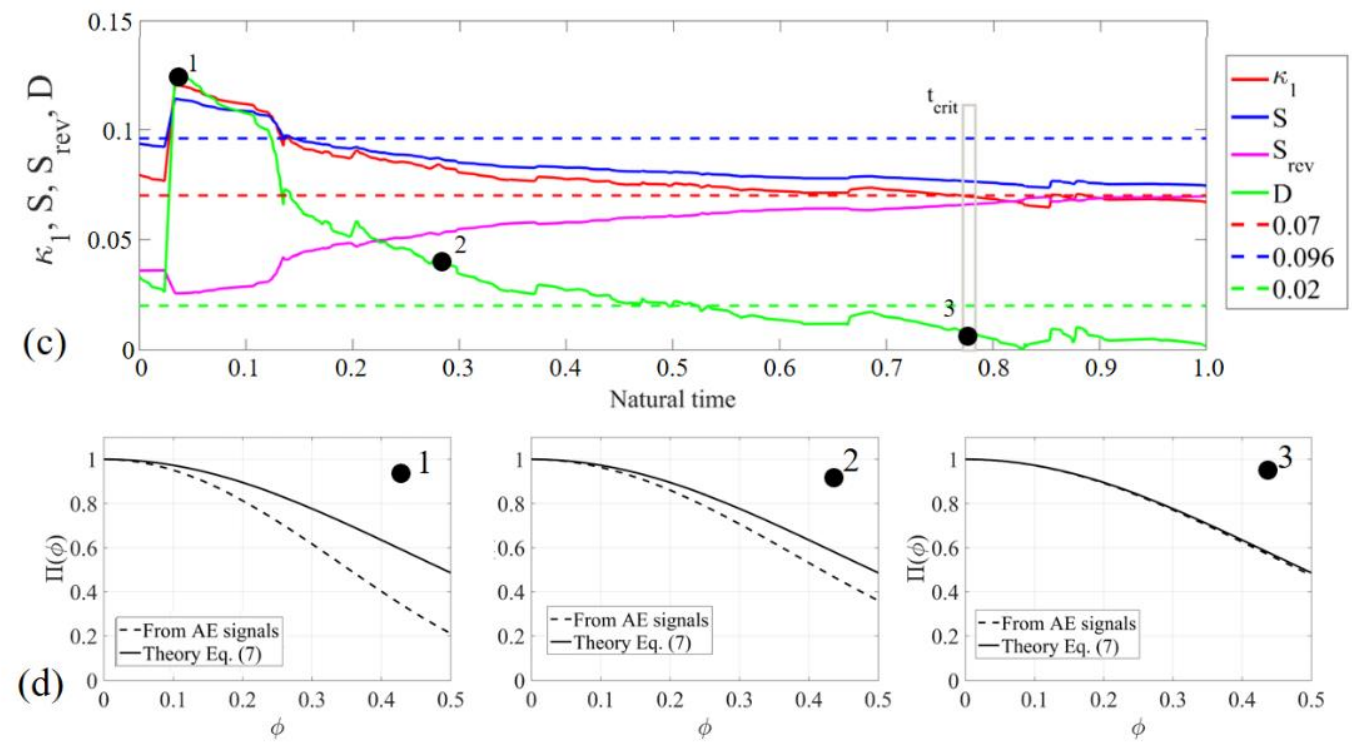

Figure 5. Applying the natural-time method: (a) a typical time series from AE signals; (b) series description in the natural time domain; (c) evolution of order parameters and entropies in natural time. $t_{\text {crit }}$ indicates the point of convergence of the criteria, where the structure enters a critical stage; (d) evolution of the normalized power spectrum until reaching $t_{\text {crit. }}$

\section{Applications}

\subsection{Three-point bending test of a glass fiber reinforced polymeric plate}

\subsubsection{Test description}

The first AE test was performed with a glass fiber reinforced polymer (GFRP) specimen. GFRP is a resin compound with a glass fiber second layer, which provides high resistance to traction, flexion, and impact. There are several ways to manufacture GFRP specimens, see Ref. [61]. The one used here was laminated manually with fibers randomly 
arranged within its matrix, Figure 6a, but some of its parts have their fibers distributed along a preferential direction. This arrangement is similar to the one used by the company "IMAP Indústria e Comércio" [62] in the making of suspended-work baskets (Figure 6b). The material's relevant properties are: modulus of elasticity $E=74.5 \mathrm{GPa}$ and mass density $\rho=1450.3 \mathrm{~kg} / \mathrm{m}^{3}$.

The specimen was subjected to a three-point bending test (TPB). Figure $6 \mathrm{c}$ shows the test configuration, including the position of the piezoelectric sensor for acquiring the AE signals. For detailed information regarding the data acquisition for this application, refer to Friedrich et al., 2020 [29]. Figure 6d shows the rupture configuration of the tested specimen, with a detailed view of the fractured region.

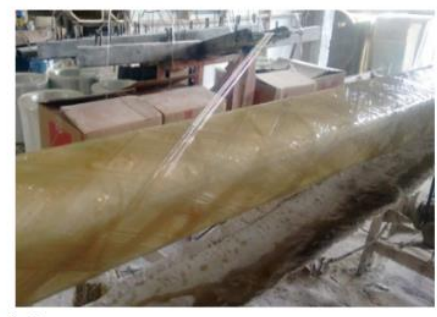

(a)

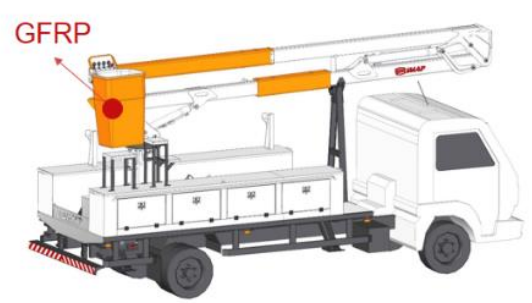

(b)

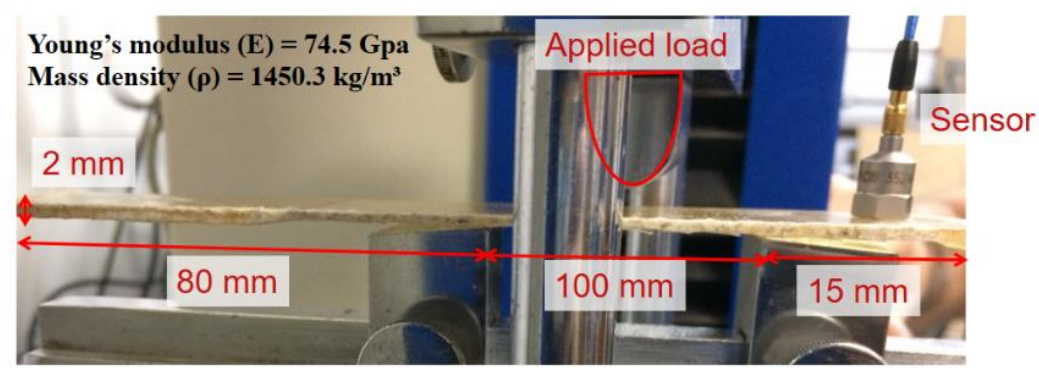

(c)

(d)

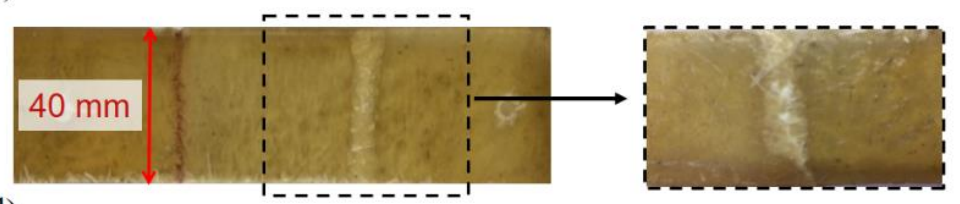

Figure 6. (a) Specimen manufacturing; (b) GFRP application example [61]; (c) Sample dimensions and test conditions; (d) Ruptured specimen final configuration with a detailed view of the fracture.

\subsubsection{Results}

Figure 7a depicts AE activity during the TPB test in normalized time (time/time peak, where time peak $=72 \mathrm{~s}$ is the peak loading time). Approximately 5050 signals above a 0.25 $\mathrm{mV}$ threshold level were separated throughout the TPB test according to the methodology described in [29]. The number of instantaneous and accumulated signals are presented in Figure $7 \mathrm{~b}$, accompanied by the evolution of the load and the AE energy, which was computed as recommended in RILEM [63]. The results in the same figure indicate a considerable correlation between the AE energy and the number of accumulated signals. In addition, one identifies three distinct patterns throughout the test. The first occurs in the region $0-0.26$, where a few sparse avalanches are identified. Their isolated nature suggests that they are probably caused by local effects, such as the specimen accommodation on its supports, without significant change in the specimen stiffness. Within the 0.26-0.78 range, damage grows indistinctly throughout the structure, with low-amplitude AE signals from no particularly discernible source. Finally, cracking coalescence takes place in the region 0.78-1.0. This is indicated by the increment in the signal avalanches, which can be considered a precursor to the imminent collapse in the structure. 
(a)

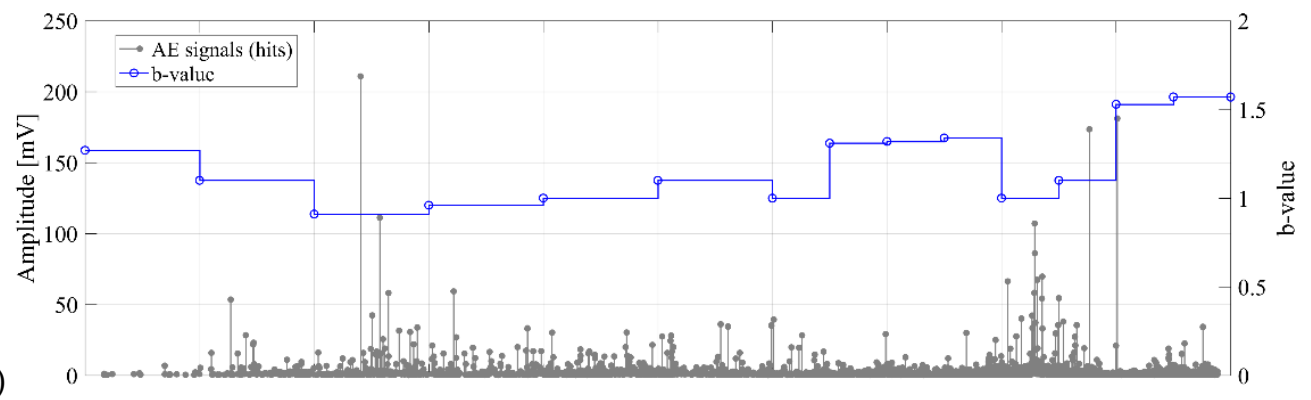

(b)
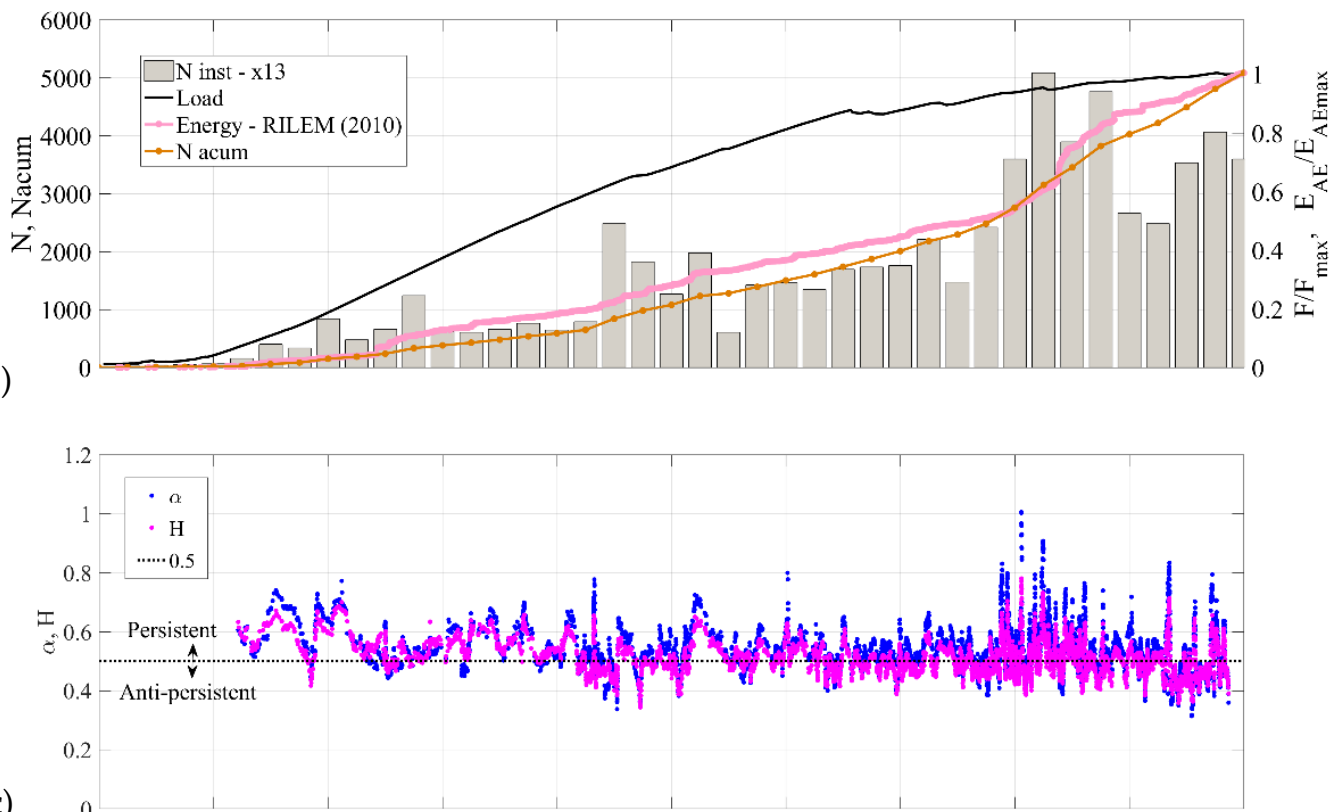

(c)

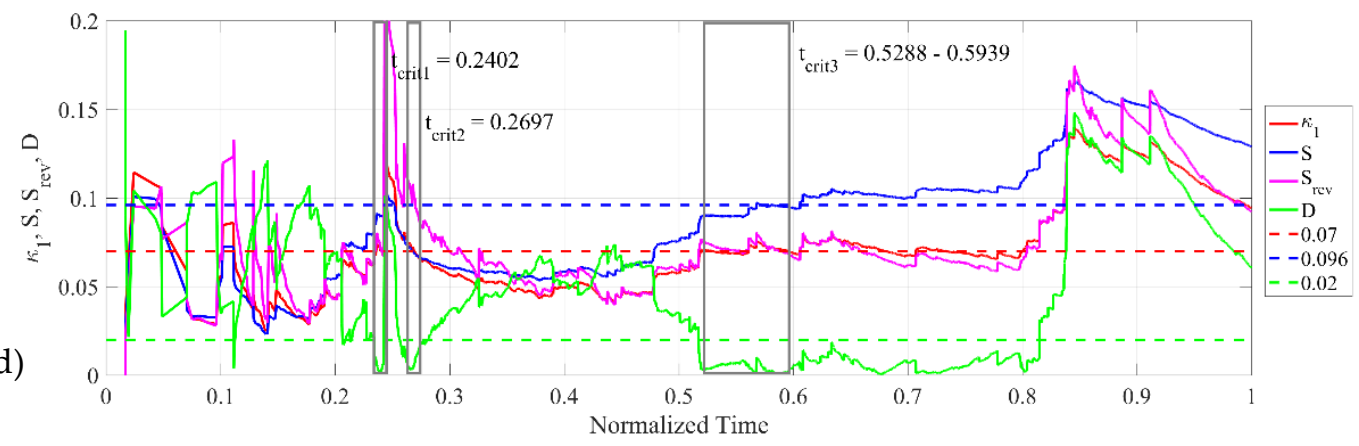

Figure 7. (a) AE signals with $b$-value analysis; (b) Number of instantaneous signals, number of accumulated signals, applied load, and accumulated energy versus normalized time. The first was multiplied by 13 to maintain the same vertical scale for instantaneous and accumulated signals; (c) Application of Hurst and DFA methods to the AE data; (d) NT analysis of the AE time series with threshold values $\left(A_{\max }^{1.5}\right)_{T h}=0.25 \mathrm{mV}$. The following auxiliary horizontal lines were added: $\kappa_{1}=0.070$ (dashed red), $\langle D\rangle$ limit $=10^{-2}$ (dashed green), entropy limit $S u \approx 0.0966$ (dashed blue).

The $b$-value is the reference parameter to the analyses performed through all methods considered in this work, and its calculation is described in Figure 7a. The test period was divided into 14 intervals, and the $b$-value was calculated for each of them [29]. Instead of repeating the expected pattern shown in Figure 1, it decreases locally in several opportunities as large-amplitude AE signals occur throughout the process. That suggests that the rupture happens in spasms uniformly distributed along the whole process instead of 
abruptly, i.e., the energy dissipates in several small events instead of being stored to a large amount and released at once in an explosive collapse.

Figure 7c summarizes the corresponding AE signal long-range analysis according to the DFA and R/S methods. The DFA (blue points) and Hurst (pink points) exponents are evaluated for each event throughout the normalized test period, considering a $25-\mathrm{hit}$ moving window, i.e., parameters are calculated at hit $\# 25$, based on a temporal series starting at hit \#1, then from $\# 2$ to $\# 26$, \#3 to \#27 and so on. The dotted black transversal line separates the values of $\alpha$ and $H$ indicating correlated/persistent signals $(>0.5)$ from noncorrelated/anti-persistent ones $(>0.5)$. The responses from both methods are similar. The persistence they indicate at the beginning of the test is probably related to the specimen accommodation on its supports, as already pointed out in Figure 7a. Then, both exponents tend to about 0.5 until the normalized time 0.75 is reached. At this instant, both exponents present significant fluctuations, which indicates the spasmodic behavior of the structure. That agrees with the findings in Skordas et al., 2020 [64], where the DFA method yielded exponents of about 0.6 (persistent behavior) for seismic signals acquired when large earthquakes were about to occur, then tending to 0.5 (random) after they occurred.

The evolution of natural-time parameters versus normalized time is plotted in Figure $7 \mathrm{~d}$. For this analysis, we follow the same reasoning as [50] and [65], taking the energy component $\left(Q_{k}\right)$ as equal to $A_{\max , k} 1.5$, where $A_{\max , k}$ is the maximum amplitude of the signal, an approach commonly found also in seismology studies [66]. Calculation of $A_{\max }{ }^{1.5}(t)$ (with $t$ denoting the conventional time) was performed hit by hit, i.e., every time a new signal comes up, a new signal is included in the NT analysis, leading to the rescaling of the $\left(\chi_{k}, Q_{k}\right)$ time series and the recalculation of $\kappa_{1}, S, S_{r e v}$ and $\langle D\rangle$. Results are shown in normalized time for easier identification of the possible entry point to a critical state, that is, the instant when criticality conditions described in section $2.2[67-68]$ are fulfilled. Three such points are indicated in Figure $7 \mathrm{~d}$. The first one occurs right before a dense region of $\mathrm{AE}$ events, at normalized time 0.24 , with an abrupt change in the AE energy rate (see Figure $7 \mathrm{~b}$ ). The second is probably related to the consecutive avalanches observed between normalized instants 0.42 and 0.52 (notice the increase of instantaneous hits in Figure $7 b)$. Finally, there is a third and more extensive period $(0.5288-0.5939)$ where parameters converge, which indicates the imminent structure collapse.

\subsubsection{Discussion}

(i) Considering the DFA and Hurst parameters, there is no discernible parameter trend in Figure $7 \mathrm{c}$ to indicate criticality. These parameters' behavior can be clarified with the aid of Figure 8, illustrating the analysis of exponents $\alpha$ and $H$ for two signal samples, for instants 0.24 and 0.82 . There are two discernible regions for each data set, and blue lines show their corresponding linear fits. However, this effect is masked if a single fit is calculated for the whole data set (red line). This is a crucial difference, because the two regions indicate different behaviors concerning time correlation: short-term correlations are persistent, whereas long-term ones are anti-persistent. Similar effects can be found in other works, such as Varotsos et al., 2003 [57], Lin et al., 2014 [69], and Silva et al., 2004 [70]. 

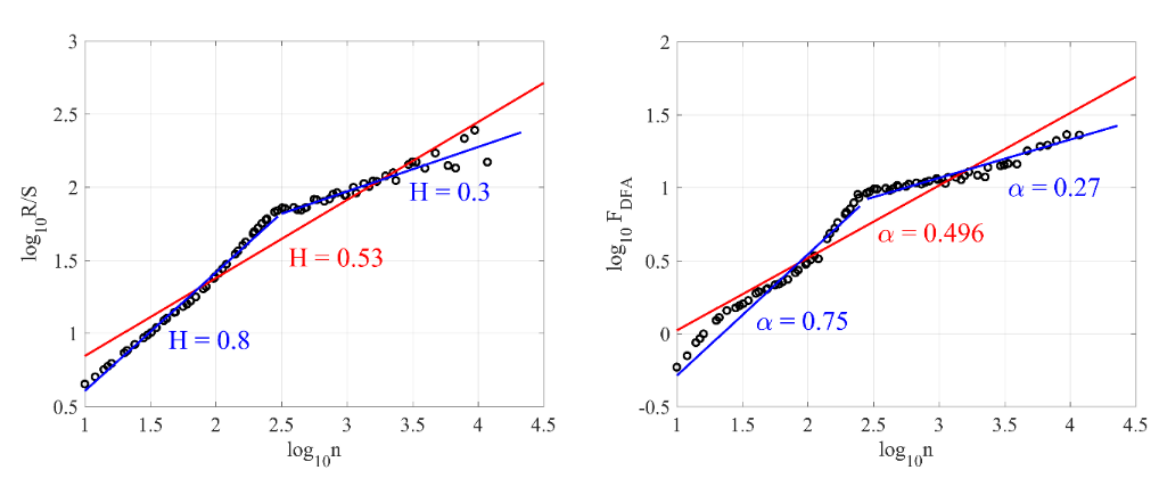

(a)
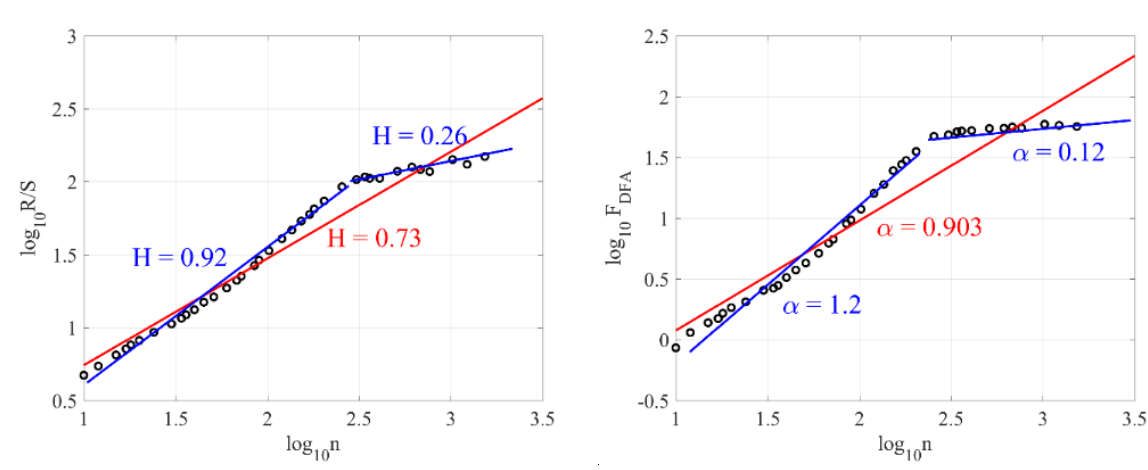

(b)

Figure 8. Details of the Hurst and DFA exponents for (a) signal at normalized time 0.24 ; (b) signal at normalized time 0.82.

To further explore this behavior, $\alpha$ was re-evaluated for short-term windows $(n<$ 300) and long-term ones $(n \geq 300)$, respectively designated $\alpha_{\text {short }}$ and $\alpha_{\text {high }}$. Corresponding results are depicted in Figure 9, where a 100-point moving mean was added to (dotted line) improve tendency visualization in each region.

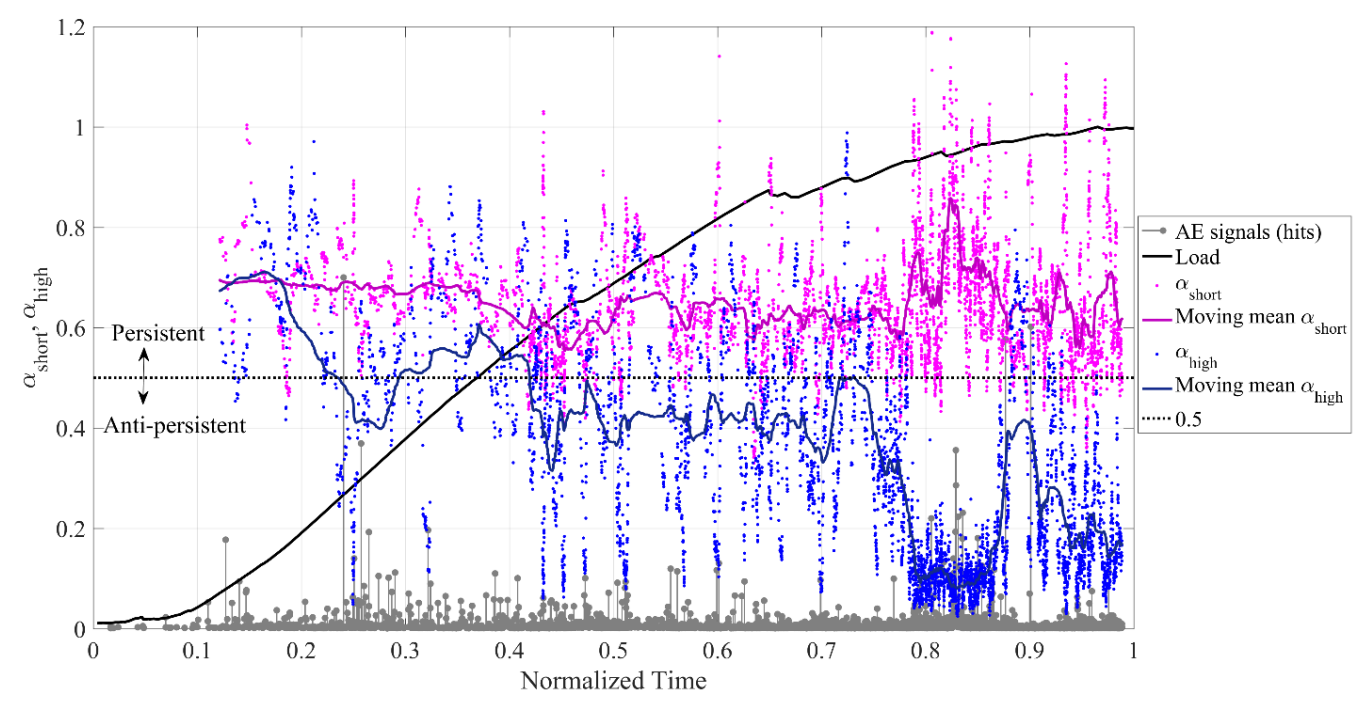

Figure 9. DFA considering short-term $\left(\alpha_{\text {shor }}\right)$ and long-term $\left(\alpha_{\text {high }}\right)$ windows for the GFRP plate. Load and AE signals out of scale. 
The correlation force for short-term windows tends to be persistent throughout the entire test, increasing significantly between normalized instants 0.78 and 0.88 , which correspond to intense AE activity as the structure approaches collapse. During the same period, long-term correlation forces are nearly nil. This opposite convergence of $\alpha_{\text {short }}$ and $\alpha_{\text {high }}$ may indicate spasmodic behavior. A similar effect is also observed between instants 0 and 0.24: initially, both $\alpha_{\text {short }}$ and $\alpha_{\text {high }}$ have an approximated average of 0.7 until normalized instant 0.18 . Then, $\alpha_{\text {high }}$ falls within the noncorrelated range $(<0.5)$ that precedes an avalanche (see Figure 7a) with large-amplitude signals.

(ii) Regarding the natural time parameters presented in Figure $7 \mathrm{~d}$, it is necessary to check whether the identified critical points represent "true coincidence," i.e., not only are all criteria satisfied, but they are also invariant to the selected threshold. Applying the same methodology described in the previous section, two new threshold values are applied to separate hits from the overall AE signals: those with amplitudes higher than (A) $1 \mathrm{mV}$ and (B) $2 \mathrm{mV}$. In the first case, the reconstructed time series comprises 2832 events, whereas the second one has 1164 . The corresponding results in natural time are given in Figure 10. Comparing with Figure 7d, two critical points are confirmed for all thresholds: the ones approximately at normalized times 0.27 and 0.59 . As for the third possibly critical point identified in Figure 7c, it is not an actual critical point since it does not appear for the other two thresholds.

As mentioned earlier, the first critical state (0.27) is related to a dense region of AE signals between 0.42 to 0.52 of the normalized time, see Figure $7 \mathrm{a}, \mathrm{b}$, where the stiffness of the structure is affected as shown by the instability present in the load curve (see Figure 7a). The second point (0.59) indicates the critical stage preceding structure failure, which occurs about 30 seconds later. Additionally, $\kappa_{1}$ tends to stabilize at nearly 0.07 for normalized time 0.6, regardless of the selected three thresholds value for hit identification. That means that the system has started to self-organize, an obvious prerequisite for failure [71].

(a)

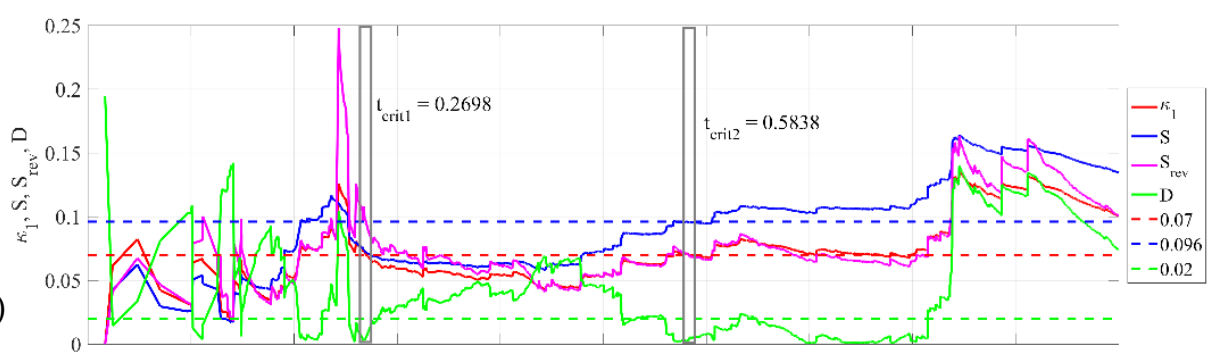

(b)

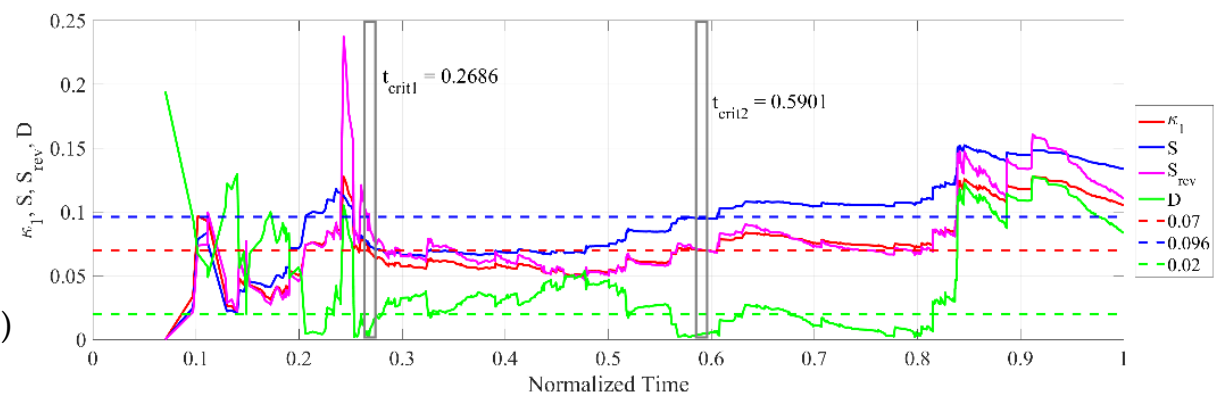

Figure 10. NT analysis of the AE time series for two threshold values $\left(A_{\max }{ }^{1.5}\right)_{T h}:(\mathbf{a}) 1.0 \mathrm{mV}$ and (b) $2.0 \mathrm{mV}$. For ease of interpreting the results, the values for $\kappa_{1}=0.070$ (dashed red), $\langle D\rangle$ limit $=10^{-2}$ (dashed green), entropy limit $S u \approx 0.0966$ (dashed blue).are also depicted as horizontal lines. 
A second approach to performing the NT analysis is to consider $Q_{k}$ that equals the acoustic emission energy $E_{A E, k}$, as suggested in RILEM [63] and shown in Figure 11 for the original data set (5050 signals, $0.25 \mathrm{mV}$ threshold). Since the methodology for calculating the NT parameters is the same as in the case of Figure 10, these new data are presented according to the same standards.

In this case, we have four points of true coincidence. The first and second critical points precede the occurrence of large avalanches, that is, regions of considerable AE activity. The third point at 0.31 again indicates the approximation of a critical region $(0.42$, 0.52 ) of the normalized time, as already mentioned in the previous approach. Finally, the last point at 0.785 clearly represents the structure's entry into a critical pre-failure stage. Thus, the analysis of natural time using the AE signal energy also presents as a valid amount of energy $(Q)$ to find the critical stages of the structure monitored by $\mathrm{AE}$ in the NT domain.

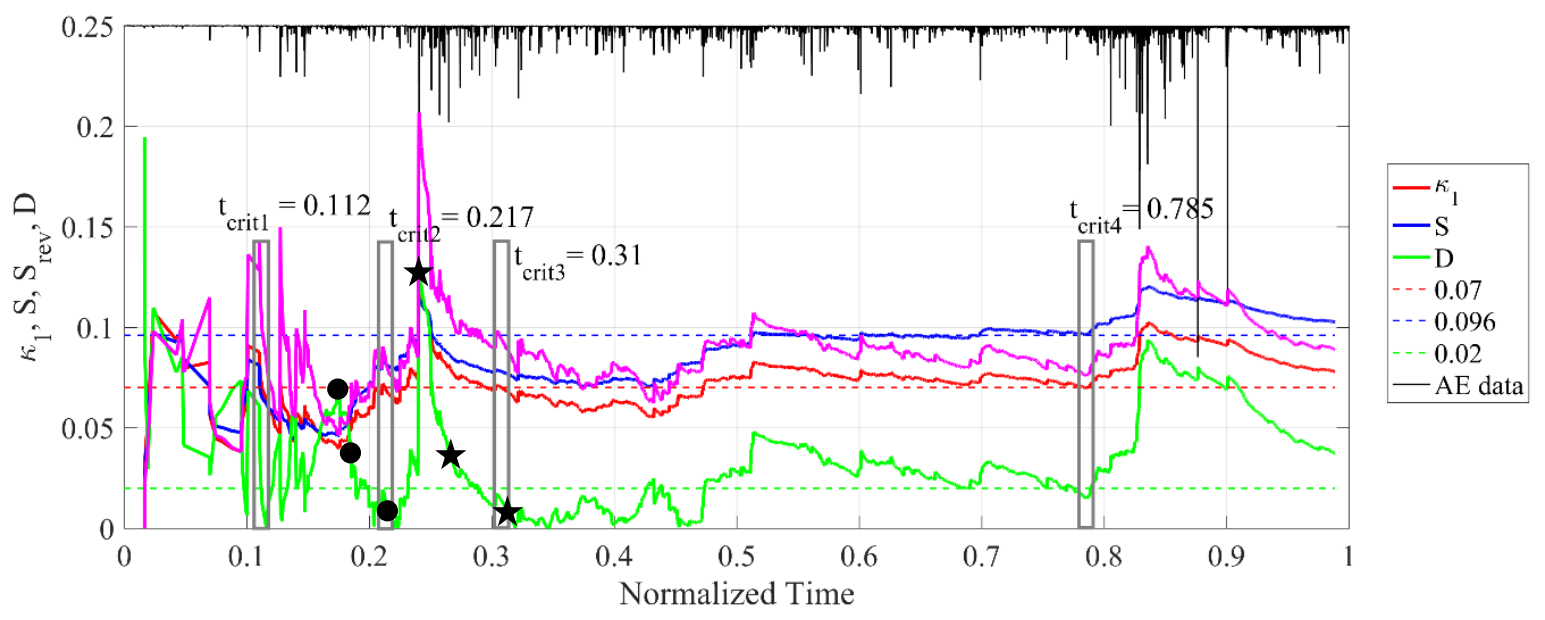

Figure 11. Natural time analysis of the AE events during the three-point bending test on the GFRP plate considering $Q_{k}=E_{A E, k}$.

In Figure 11, the true coincidence points 2 and 3 were monitored to evaluate the behavior of the normalized power spectra $\Pi(\phi)$ versus the natural frequency $(\phi)$ relationship obtained by analyzing the series of signals, in comparison with the ideal estimate by Equation (7). For each point, we analyzed three normalized time instants, prior to the critical time being reached, black circles and black stars, respectively. It can be seen in the results presented in Figure 12, that when approaching the point of true coincidence, the curves obtained from the series of AE signals, and those theoretically determined, approach from the bottom to the top. At the same time if we look at Figure 11, we notice that the value of $\kappa_{1}$ also approaches to 0.07 , so both order parameters for criticality converge.

\section{2. "Spaghetti" Bridge Model}

\subsubsection{Test description}

The same analysis methods proposed here were applied to another AE data set, obtained from the spaghetti bridge depicted in Figure 13a. This bridge was built in 2018 by undergraduate engineering students to take part in the contest at the Engineering School of Federal University of Rio Grande do Sul (UFRGS), which has occurred twice a year since 2004. Detailed information regarding the contest is given in [72], and all appropriate steps for acquiring the corresponding AE data are described in Tanzi et al., 2021 [30]. 

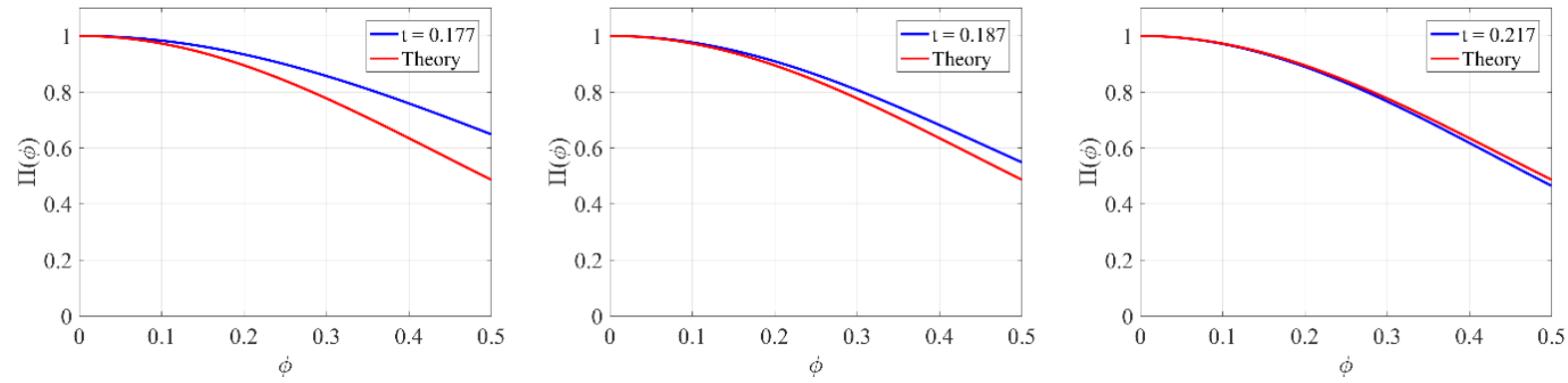

(a)
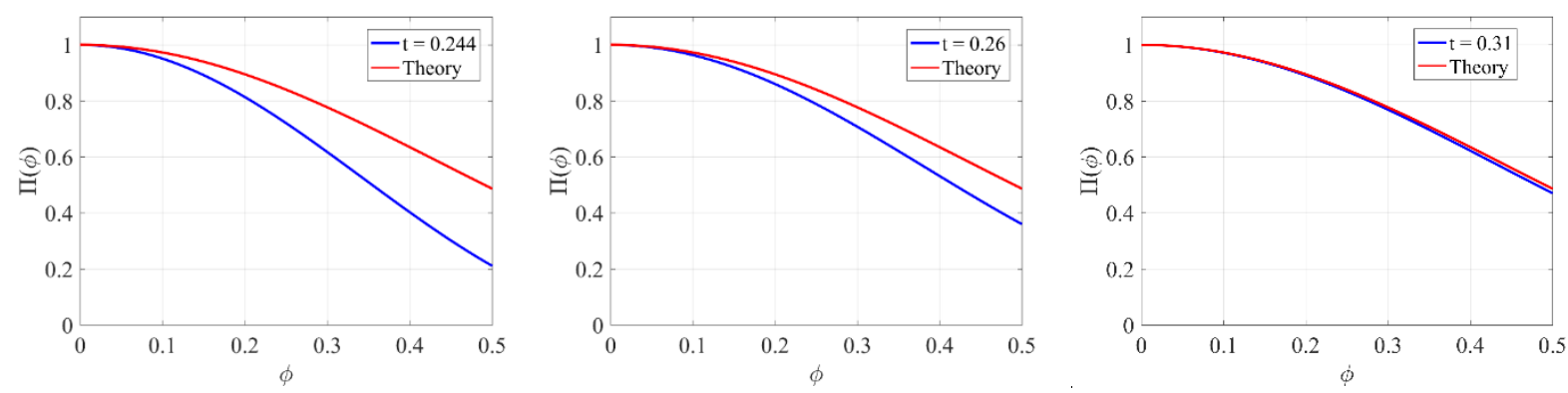

(b)

Figure 12. Evolution of $\Pi(\phi)$ of the AE activity, (a) critical time 2 (black circles $-t_{\text {crit2 }}=0.217$ ) and, (b) critical time 3 (black stars $-t_{\text {crit }}=0.31$ ).

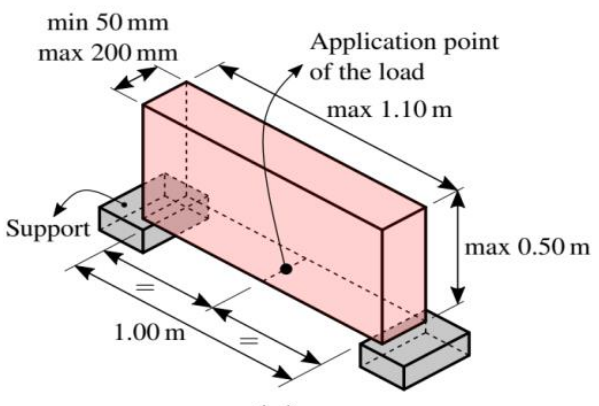

(a)

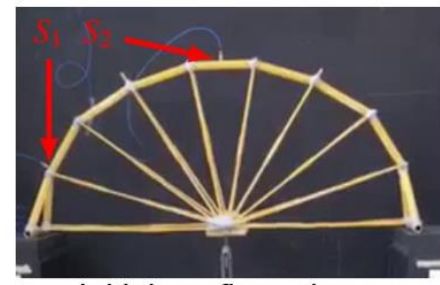

Initial configuration

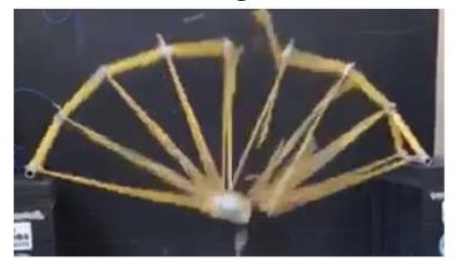

Rupture configuration

Figure 13. "Spaghetti" Bridge Model: (a) Geometric restrictions for the contest [72], (b) Initial and final configurations of the bridge during the test.

\subsubsection{Results}

The "Spaghetti" Bridge Model results throughout the loading test are shown in Figure 14. For a threshold level of $0.7 \mathrm{mV}$, the AE sensors detected 230 signals, Figure 14a. These results appear in Figure $14 \mathrm{~b}$ as the numbers of instantaneous and accumulated signals, the loading evolution, and the acoustic emission energy against normalized time. For this test, signals occur nearly when the load increases, indicating that their distribution depends explicitly on the loading pattern. Additionally, up to $t \approx 0.6$, signal counts grow at an approximately constant rate. Then, there is a sharp increase in the number of signals for a brief period. After that, signal counts grow once again at a nearly constant rate but a faster pace. That agrees with the expected general behavior for AE-based analysis 
methods, and this work seeks to confirm such a conclusion through the methods presented in Section 2, as summarized in Figure 14.

The $b$-value parameter (Figure 14a) was calculated in [30] by separating the data set into packs of 25 signals, with five-signal overlaps between successive packs. The sudden coefficient changes at $t \approx 0.6$ coincide with the avalanche observed in the signals count, confirming this point as critical for the test in question.

(a)

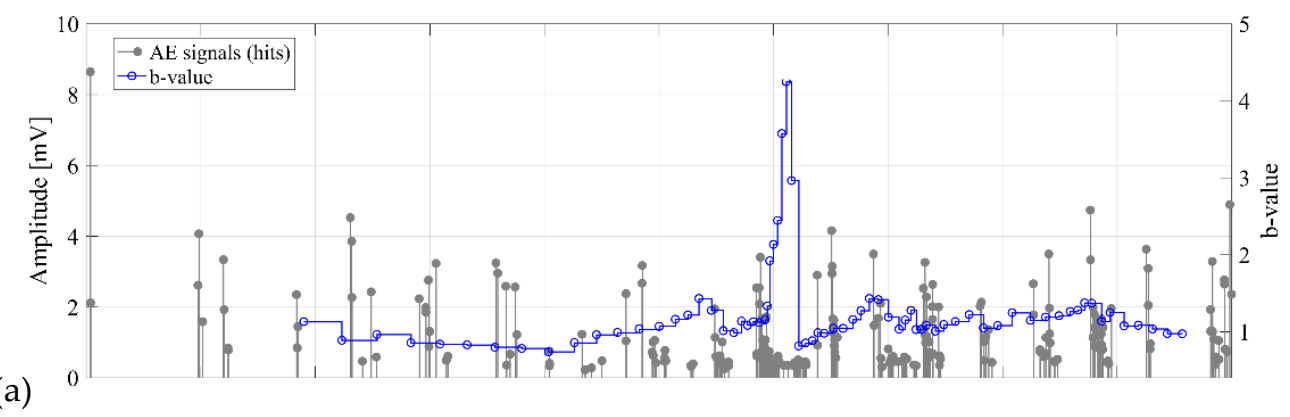

(b)
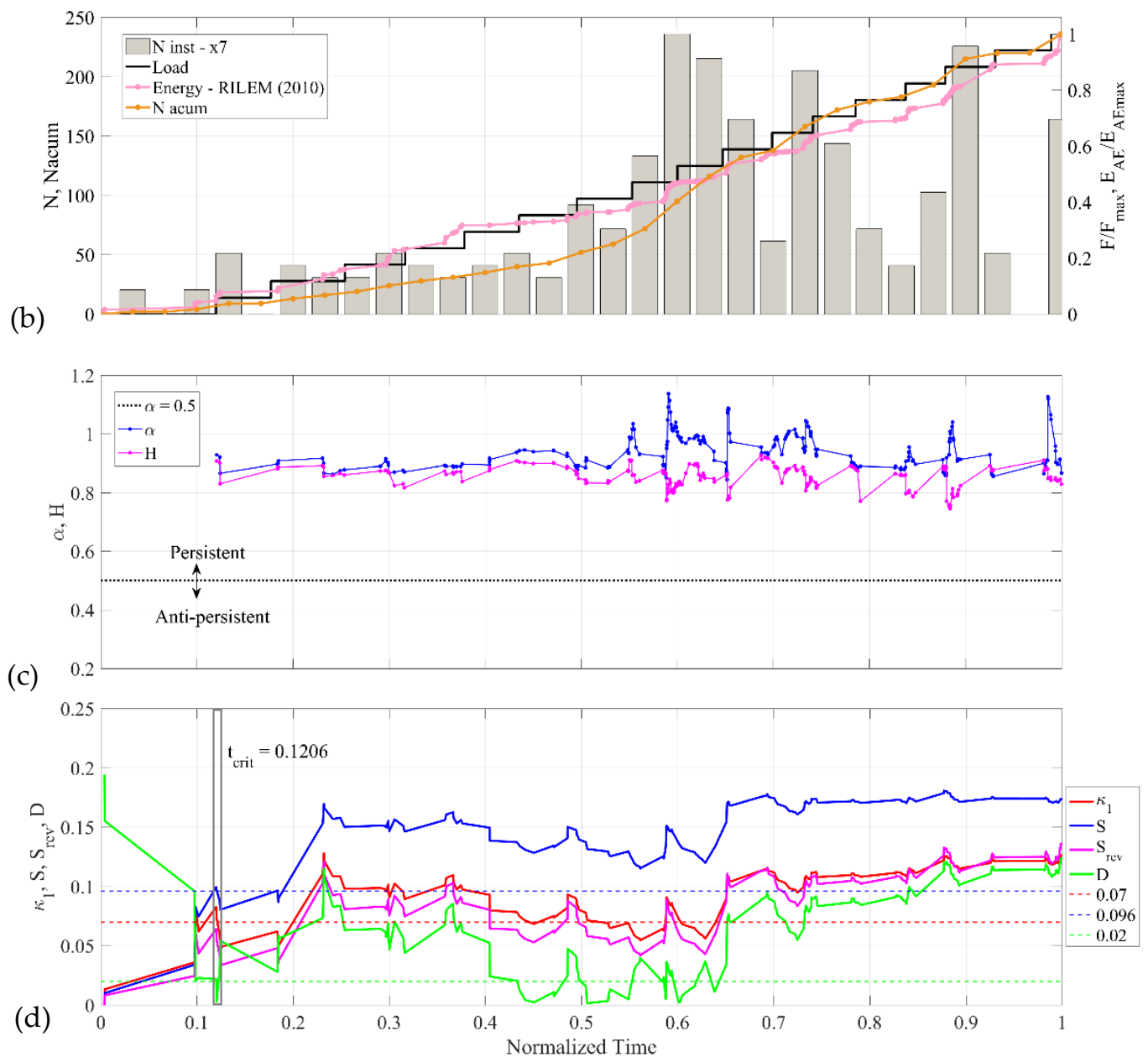

Figure 14. (a) AE signals and corresponding $b$-value for the spaghetti bridge loading test, as described in [30]); (b) Numbers of instantaneous signals, number of accumulated signals, applied load, and accumulated energy versus normalized time. The first was multiplied by 7 to maintain the same vertical scale for instantaneous and accumulated signals; (c) application of Hurst and DFA methods to the AE data; (d) NT analysis of the AE time series (this figure's format follows that of Figure $7 d)$.

The long-range correlation results for the spaghetti bridge appear in Figure 14c. The calculation methodology is the same one used with the GFRP plate, but since this test has yielded fewer signal samples, a 6-signals moving window was used to compute $\alpha$ and 
$H$. Both parameters behave similarly until $t \approx 0.55$, with values of about 0.9 indicating persistent correlations. Then, they present significant local changes, most notably at normalized instants $0.6,0.65$, and 0.79 , with the Hurst exponent appearing as more sensitive to the observed signal changes. Contrary to the GFRP plate case, no anti-persistent correlations are observed, just strong long-range correlations with local instabilities for the points of more significant AE activity.

Finally, the results for natural time analysis are presented in Figure 14d, considering $Q_{k}=A_{\max , k}^{1.5}$ and using the same calculation method described for the GFRP plate. A single coincidence point is identified at normalized time 0.1206 . If only the order parameters for criticality $\left(\kappa_{1}\right.$ and $\left.\langle D\rangle\right)$ are considered, new convergence points are observed at the normalized instant 0.54 , which is close to the already discussed sudden rise in AE energy at $t \approx 0.6$ (Figure $14 \mathrm{~b}$ ), which was also identified through the $b$-value (Figure 14a). The last convergence point occurs at $t=0.62$, where the two exponents change behavior again until the structure collapses. Subsequent analysis can show that these results do not change with respect to the amplitude threshold for identifying hits. Contrary to the GFRP plate, however, there is no discernible coincidence point in the vicinity of the structure's failure.

\subsubsection{Discussion}

(i) As in the first application, important information was omitted during the first calculation of parameters $H$ and $\alpha$. For this reason, such a calculation was repeated for different time scales to further evaluate these parameters' usefulness in monitoring structure damage.
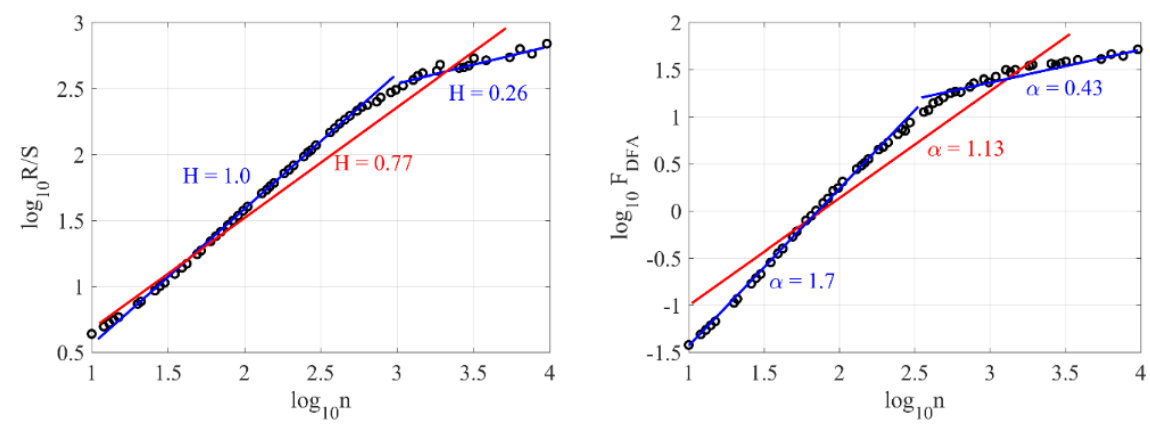

(a)
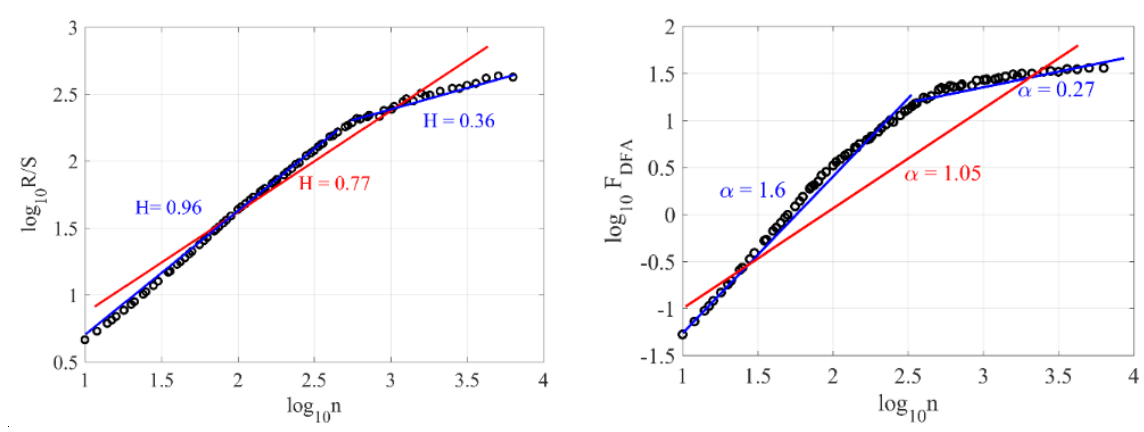

(b)

Figure 15. Details of the Hurst and DFA exponents for (a) signal \#84 at normalized time 0.59; (b) signal \#127 at normalized time 0.65 .

Figure 15 illustrates $H$ and $\alpha$ for signals identified at normalized instants 0.59 and 0.65. The red line indicates the overall linear fit for the data, whereas the fits for each subset are shown in blue. The values for $H$ and $\alpha$ are high for short-time ranges, i.e., high- 
frequency signals are persistent as expected from [51], whereas low-frequency ones $(n>=$ 400) are anti-persistent.

Naming the DFA values for $n<400$ e $n>=400$ as $\alpha_{\text {shorr }}$ and $\alpha_{\text {high }}$ respectively, their behavior throughout time appears in Figure 16, where AE signals' amplitudes are out of scale. As in the first application, these parameters have opposite tendencies as a high-AEactivity point approaches. While high-frequency correlations are always persistent, lowfrequency ones are persistent for noncritical periods, turning into anti-persistent at critical points, such as at normalized instant 0.6 and during the subsequent avalanches.

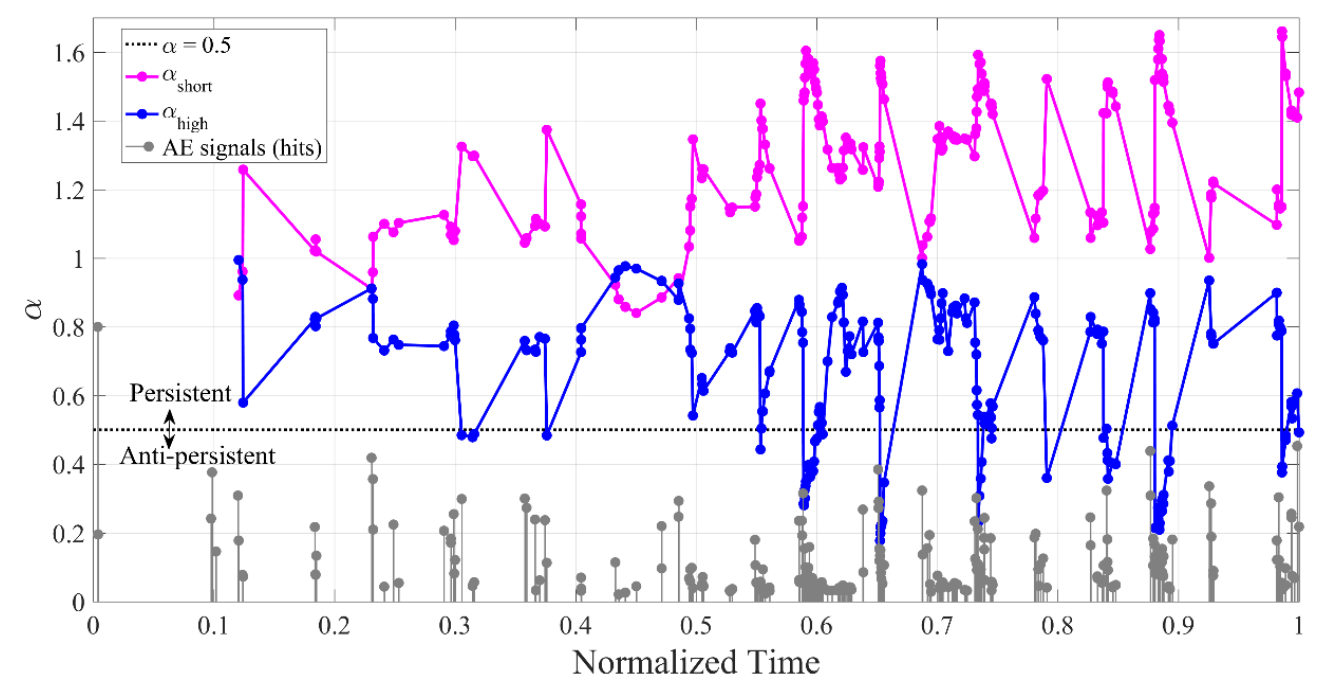

Figure 16. DFA results considering short $\left(\alpha_{\text {short }}\right)$ and large $\left(\alpha_{\text {high }}\right)$ time ranges for the spaghetti bridge. AE signals out of scale.

(ii) The natural time analysis of the spaghetti bridge's AE results was also performed using $Q_{k}=E_{A E, k}$, as shown in Figure 17a. Now, two possibly critical points are identified, one at $t=0.4046$ and the other at $t=0.6025$, where the latter is followed by significant changes in both the order parameters and the two entropy metrics, anticipating the bridge collapse. Figure $17 \mathrm{~b}$ depicts the normalized power spectrum $\Pi(\phi)$ versus natural frequency $(\phi)$ calculated for the AE signal (blue), compared to its expected theoretical value (red). Once again, the criticality parameter $\Pi(\phi)$ appears as a good predictor of critical stages throughout the test.

\section{Conclusions}

This work focused on using time series methods to analyze acoustic emission data from two experimental tests with different materials: i) a fiber-reinforced polymer plate undergoing a three-point bending test; ii) a spaghetti bridge model from an undergraduate-level student loading contest. The time series analysis was carried out through two approaches: calculating DFA and Hurst exponents to characterize long-range correlations and analysis in the natural time domain. The purpose was to identify the possibility of using these methodologies to predict critical points or failure in the studied structures. From the results, one concludes that:

- Both Hurst and DFA exponents yielded very similar results, which indicate that no high-order trends are present in the data. 


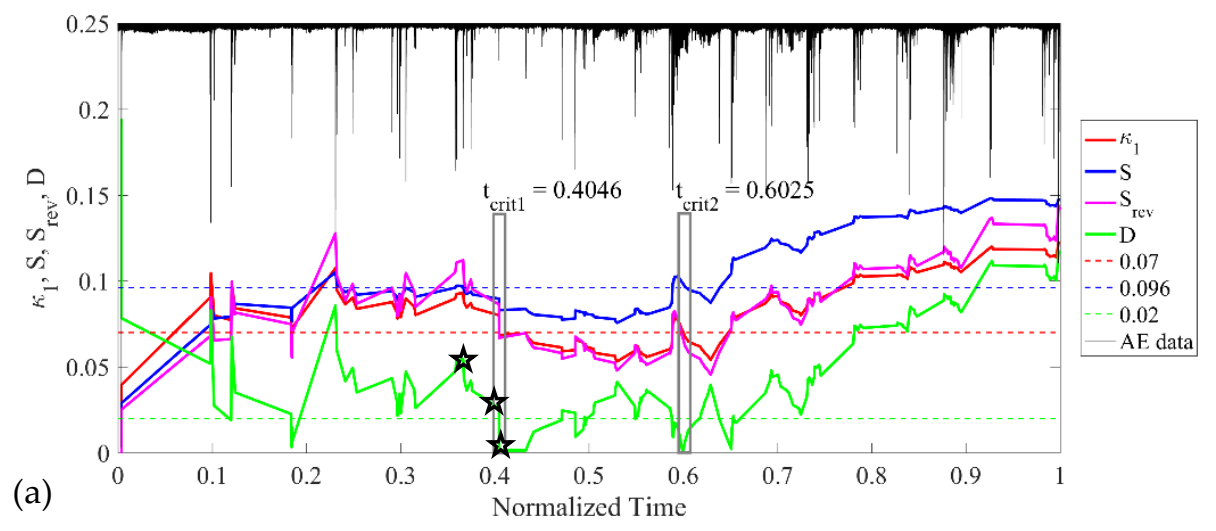

(b)
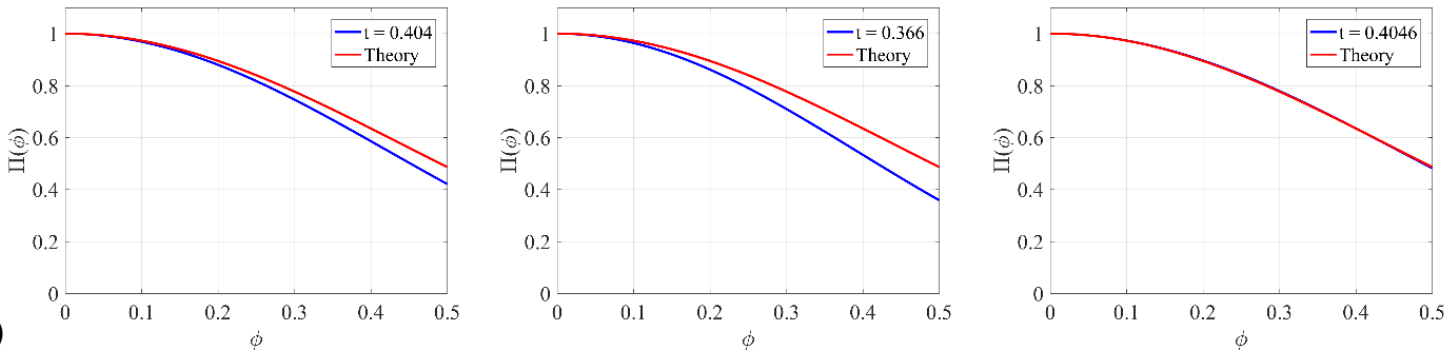

Figure 17. (a) Results from the natural time analysis of the AE events during the loading test for the spaghetti model bridge, according to the same format described for Figure 7d. (b) Evolution of $\Pi(\phi)$ during the same test.

- For both applications, the DFA parameter calculated for the whole data set was inconclusive. However, when considered separately, the low $\left(\alpha_{\text {low }}\right)$ and high $\left(\alpha_{\text {high }}\right)$ state levels indicated a critical region where one converged to extreme anti-persistent correlations, and the other, to extreme persistent ones. That highlights these parameters' usefulness in indicating the criticality of the structure.

- The analysis in the natural time domain showed that the convergence of the order parameters and the entropies could predict the structure's entry in a critical stage, whether it is calculated from the AE energy or from counting the ruptured samples.

Author Contributions: Conceptualization, L.F.F., A.B.C., E.C., I.I., G.N. and G.L.; Methodology, L.F.F., E.C., B.N.R.T., I.I and G.L.; Formal analysis, L.F.F, E.C, I.I.; Investigation, L.F.F, E.C., A.B.C, B.N.R.T. and L.E.K.; Writing-original draft preparation, L.F.F, A.B.C, E.C, I.I. and M.S.; Writingreview and editing, L.F.F, B.N.R.T., I.I., L.E.K., G.L., G.N., M.S.; Visualization, L.F.F., I.I.; Supervision, L.E.K., G.N., G.L., I.I.; All authors have read and agreed to the published version of the manuscript.

Funding: This research was funded by CNPq and CAPES (Brazil), and the sponsorship guaranteed with basic research funds provided by Politecnico di Torino (Italy).

Acknowledgments: The authors wish to acknowledge the Brazilian National Council for Scientific and Technological Development (CNPq), the Coordination for the Improvement of Higher Level of Education Personnel (CAPES) and the sponsorship guaranteed with basic research funds provided by Politecnico di Torino, Italy for their financial aids in this work.

Conflicts of Interest: The authors declare no conflict of interest. 


\section{Nomenclature:}

Signal amplitude

$A_{\max } \quad$ Maximum signal amplitude

$b \quad b$-value parameter

$D, d \quad$ Fractal dimension

$F_{D F A} \quad$ Average fluctuations in DFA

n

Window size

$\alpha$

$N_{\max }$

Total number of points in the series

A time series

$y$

Integrate the time series

$X$

$R$

S

$n w$

$H \quad$ Hurst exponent

$n_{\text {min }}$
Sum the differences from the mean in Hurst analysis

Local range

Standard deviation

Total number of windows

Smallest window size $n_{\max }$

$\chi$

Natural time

$Q$

Energy of the individual event

Normalized energy $(Q)$

$\kappa$

$S$

Entropy in natural time

$S_{r e v} \quad$ Time-reversal entropy

$\omega$

Natural angular frequency

$\phi \quad$ Frequency in natural time

$\Pi \quad$ Normalized power spectrum

$S_{u} \quad$ Entropy of uniform noise

E Young's modulus

$\rho \quad$ Mass density

$\alpha_{\text {short }} \quad$ Short-term windows

$\alpha_{\text {high }} \quad$ Long-term windows 


\section{References}

1. Nemat-Nasser, S.; Hori, M. Micromechanics: overall properties of heterogeneous materials, North-Holland Series in Applied Mathematics and Mechanics, 1999.

2. Torquato, S. Random Heterogeneous Materials: Microstructure and Macroscopic Properties; Springer New York, 2013.

3. Gross, D.; Seelig, T. Fracture Mechanics - With an Introduction to Micromechanics, Mechanical Engineering Series, Springer Germany, 2006.

4. Kachanov, L. Time of the rupture process under creep conditions. Izvestiia Akademii Nauk SSSR 1957, 8, $26-31$.

5. Chaboche, J. Continuum damage mechanics: Present state and future trends. Nucl. Eng. Des. 1987, 105, 19-33.

6. Voyiadjuis, G.Z. Handbook of Damage Mechanics, Nano to Macro Scale for materials and Structures. Springer, 2014.

7. Daniels, H. E. The statistical theory of the strength of bundles of threads. IProc. R. Soc. Lond. 1945, 405-435.

8. Hansen, A.; Hemmer, P.C.; Pradhan, S. The Fiber Bundle Model: Modeling Failure in Materials; Statistical Physics of Fracture and Breakdown; Wiley-VCH Verlag GmbH \& Co. KGaA: Weinheim, Germany, 2015; ISBN 978-3-527-41214-3

9. Jenabidehkordi, A. Computational methods for fracture in rock: a review and recent advances. Front. Struct. Civ. Eng. 2019, 13, 273-287.

10. Grosse, C.U.; Ohtsu, M. Acoustic Emission Testing. Springer Berlin Heidelberg, 2008.

11. Aki, K. Scaling law of seismic spectrum. J. Geophys. Res. 1967, 72, 1217-1231.

12. Carpinteri, A.; Lacidogna, G.; Puzzi, S. From criticality to final collapse: evolution of the "b-value" from 1.5 to 1.0. Chaos Solit. 2009, 41, 843-853.

13. Kogan, S.M. Low-Frequency Current Noise with a 1/f Spectrum in Solids. Sov. Phys. Usp. 1985, 28, $170-195$.

14. Milotti, E. 1/f Noise: A Pedagogical Review. arxiv 2002, arXiv:physics/0204033.

15. Carpinteri, A.; Lacidogna, G.; Accornero, F. Fluctuations of 1/f Noise in Damaging Structures Analyzed by Acoustic Emission. Appl. Sci. 2018.

16. Aggelis, D.G. Classification of cracking mode in concrete by acoustic emission parameters. Mech. Res. Commun. 2011, 38, 153157.

17. Carpinteri, A.; Lacidogna, G.; Corrado, M.; Di Battista, E. Cracking and Crackling in Concrete-Like Materials: A Dynamic Energy Balance. Eng. Fract. Mech. 2016, 155, 130-144.

18. Alava, M.J.; Nukala, P.K.V.V.; Zapperi, S. Statistical models of fracture. Adv. Phys. 2006, 55, 349-476.

19. Xu, D.; Liu, P.; Chen, Z.; Cai, Q.; Leng, J. Dynamic feature evaluation on streaming acoustic emission data for adhesively bonded joints for composite wind turbine blade. Struct. Health. Monit. 2021.

20. Wilson, K.G. Problems in Physics with Many Scales of Length. Sci. Am. 1979, 241, 140-157.

21. Tainter, J.A. The Collapse of Complex Societies, New Studies in Archaeology, Cambridge Univ. Press: Cambridge; 1988. ISBN 9780-521-38673-9.

22. Rosser, J.B. The Rise and Fall of Catastrophe Theory Applications in Economics: Was the Baby Thrown Out with the Bathwater. J. Econ. Dyn. Control 2007, 31, 3255-3280.

23. Rundle, J.B.; Turcotte, D.L.; Shcherbakov, R.; Klein, W.; Sammis, C. Statistical physics approach to understanding the multiscale dynamics of earthquake fault systems, Rev. Geophys. 2003, 1019-1041.

24. Turcotte, D.L.; Newman, W.I.; Shcherbakov, R. Micro and Macroscopic Models of Rock Fracture. Geophys. J. Int. 2003, 152, 718728.

25. Hurst, H.E. Long-term storage capacity of reservoirs. Trans. Am. Soc. Civ. Eng. 1951, 116, 770-808.

26. Peng, C.K. et al. Mosaic Organization of DNA Nucleotides, Phys. Rev. E 1994, 49, 1685-1689.

27. Varotsos, P.A.; Sarlis, N.V.; Skordas, E.S. Pract. Athens. Acad. 2001, 76, 294-321.

28. Potirakis, S.M.; Karadimitrakis, A.; Eftaxias, K. Natural time analysis of critical phenomena: The case of pre-fracture electromagnetic emissions. Chaos 2013.

29. Friedrich, L.; Colpo, A.; Maggi, A.; Becker, T.; Lacidogna, G.; Iturrioz, I. Damage process in glass fiber reinforced polymer specimens using acoustic emission technique with low frequency acquisition. Compos. Struct. 2021, 256, 113105, doi:10.1016/j.compstruct.2020.113105.

30. Tanzi, R.B.N.; Sobczyk, M.; Becker, T.; Segovia González, L.A.; Vantadori, S.; Iturrioz, I.; Lacidogna, G. Damage Evolution Analysis in a "Spaghetti" Bridge Model Using the Acoustic Emission Technique. Appl. Sci. 2021, 11, 2718. https://doi.org/10.3390/app11062718.

31. Sagasta, F.; Zitto, M.E.; Piotrkowski, R.; Benavent-Climent, A.; Suarez, E.; Gallego, A. Acoustic emission energy $b$-value for local damage evaluation in reinforced concrete structures subjected to seismic loadings. Mech. Syst. Signal Process. 2018, 102, $262-277$.

32. Richter, C.F. Elementary seismology. San Francisco and London: W.H. Freeman, 1958.

33. Carpinteri, A.; Lacidogna, G.; Niccolini, G. Fractal analysis of damage detected in concrete structural elements under loading. Chaos Soliton Fract 2009, 42, 2047-2056.

34. Varotsos, P.A.; Sarlis, N.V.; Skordas, E.S. Detrended fluctuation analysis of the magnetic and electric field variations that precede rupture. Chaos: An Interdisciplinary Journal of Nonlinear Science 2009. https://doi.org/10.1063/1.3130931

35. Kantelhardt, J.W.; Koscielny-Bunde, E.; Rego, H.H.A.; Havlin, S.; Bunde, A. Detecting long-range correlations with detrended fluctuation analysis. Phys. A: Stat. Mech. Appl. 2001, 295, 441-454.

36. Mandelbrot, B.B. The Fractal Geometry of Nature. W. H. Freeman and Company New York, 1982. 
37. Machado Filho, A.; da Silva, M.F.; Zebende, G.F. Autocorrelation and cross-correlation in time series of homicide and attempted homicide. Phys A: Stat. Mech. Appl. 2014, 400, 12-19.

38. Coronado, A.V.; Carpena, P. Size Effects on Correlation Measures. J. Biol. Phys. 2005, 31, 121-133.

39. Talkner, P.; Weber, R.O. Power spectrum and detrended fluctuation analysis: Application to daily temperatures. Phys. Rev. E 2000, 62, 150.

40. Hu, K.; Ivanov, P.C.; Chen, Z.; Carpena, P.; Stanley, H.E. Effect of trends on detrended fluctuation analysis. Phys. Rev. E Stat Nonlin. Soft. Matter. Phys. 2001.

41. Landa, E.; Morales, I.O.; Fossion, R.; Stránský, P.; Velázquez, V.; López Vieyra, J.C.; Frank, A. Criticality and long-range correlations in time series in classical and quantum systems. Phys. Rev. E 2011, doi:10.1103/PhysRevE.84.016224.

42. Mariani, M.C.; Asante, P.K.; Bhuiyan, M.A.M.; Beccar-Varela, M.P.; Jaroszewicz, S.; Tweneboah, O.K. Long-Range Correlations and Characterization of Financial and Volcanic Time Series. Mathematics 2020; 8, 441.

43. Damouras, S.; Chang, M.D.; Sejdić, E.; Chau, E. An empirical examination of detrended fluctuation analysis for gait data. Gait Posture 2010, 31, 336-340.

44. Rigoli, L.M.; Lorenz, T.; Coey, C.; et al. Co-actors Exhibit Similarity in Their Structure of Behavioural Variation That Remains Stable Across Range of Naturalistic Activities. Sci. Rep. 2020. https://doi.org/10.1038/s41598-020-63056-x.

45. Bassingthwaighte, J.B. Raymond GM. Evaluating rescaled range analysis for time series. Ann. Biomed. Eng. 1994; 432-444. https://doi.org/10.1007/BF02368250.

46. Li, J.; Chen, Y. Rescaled range $(\mathrm{R} / \mathrm{S})$ analysis on seismic activity parameters. Acta Seimol. Sin. 2001, 14, 148-155. https://doi.org/10.1007/s11589-001-0145-9.

47. Varotsos, P.A.; Sarlis, N.V.; Skordas, E.S. Long-range correlations in the electric signals that precede rupture. Phys. Rev. E 2002. doi: 10.1103/PhysRevE.66.011902.

48. Hausdorff, J.M.; Mitchell, S.L.; Firtion, R.; Peng, C-K.; Cudkowitz, M.E.; Wei, J.Y.; et al. Altered fractal dynamics of gait: reduced stride-interval correlations with aging and Huntington's disease. J. Appl. Physiol. 1997, 82, 262-269.

49. Jordan, K.; Challis, J.H.; Newell, K.M. Long range correlations in the stride interval of running. Gait Posture 2006, $24,120$.

50. Niccolini, G.; Manuello, A.; Marchis, E.; Carpinteri, A. Signal frequency distribution and natural-time analysis from acoustic emission monitoring of an arched structure in the Castle of Racconigi. Nat. Hazards Earth Syst. Sci. 2017, 1025-1032. doi: https://doi.org/10.5194/nhess-17-1025-2017

51. Varotsos, P.A.; Panayiotis, A.; Sarlis, N.V.; Skordas, E.S. Natural Time Analysis: The new view of time. Springer, 2011. doi: 10.1007/978-3-642-16449-1.

52. Ramírez-Rojas, A.; Flores-Márquez, E.L. Order parameter analysis of seismicity of the Mexican Pacific coast, Phys. A: Stat. Mech. Appl. 2013, 392, 2507-2512.

53. Shannon, C.E. A mathematical theory of communication. Bell. Syst. Tech. J. 1948, 379:423. https://doi.org/10.1145/584091 .584093.

54. Potirakis, S.M.; Mastrogiannis, D. Critical features revealed in acoustic and electromagnetic emissions during fracture experiments on LiF. Phys. A: Stat. Mech. Appl. 2017, 485, 11-12.

55. Daniel, S.H. Seismic electric signals (SES) and earthquakes: A review of an updated VAN method and competing hypotheses for SES generation and earthquake triggering. Phys. Earth Planet Inter. 2020, 302.

56. Varotsos, P.A.; Sarlis, N.V.; Skordas, E.S. Spatio-temporal complexity aspects on the interrelation between seismic electric signals and seismicity. Pract. Athens Acad. 2001, 76, 294-321.

57. Varotsos, P.A.; Panayiotis, A.; Sarlis, N.V.; Skordas, E.S. Long-range correlations in the electric signals that precede rupture: Further investigations. Phys. Rev. E 2003, doi: 10.1103/PhysRevE.67.021109.

58. Varotsos, P.A.; Tanaka, H.K.; Sarlis, N.V.; Skordas, E.S. Similarity of fluctuations in correlated systems: The case of seismicity. Phys. Rev. E 2005. doi: 10.1103/PhysRevE.72.041103

59. Varotsos, P.A.; Sarlis, N.V.; Skordas, E.S.; Tanaka, H.K.; Lazaridou, M.S. Attempt to distinguish long-range temporal correlations from the statistics of the increments by natural time analysis. Phys. Rev. E 2006, doi: 10.1103/PhysRevE.74.021123

60. Niccolini, G.; Potirakis, S.M.; Lacidogna, G., Borla, O. Criticality Hidden in Acoustic Emissions and in Changing Electrical Resistance during Fracture of Rocks and Cement-Based Materials. Materials 2020. https://doi.org/10.3390/ma13245608

61. Akovali, G. Handbook of Composite Fabrication. Rapra Technology LTDA, 2001.

62. Imap. Available online: https://imap.com.br (Accessed March 20, 2020).

63. RILEM, T.C. Recommendation of RILEM TC 212-ACD: acoustic emission and related NDE techniques for crack detection and damage evaluation in concrete: Test method for classification of active cracks in concrete structures by acoustic emission. Mater Struct 2010, 43, 1187-1189.

64. Skordas, E.S.; Christopoulos, S.R.G.; Sarlis, N.V. Detrended fluctuation analysis of seismicity and order parameter fluctuations before the M7.1 Ridgecrest earthquake. Nat Hazards 2020, 100, 697-711. https://doi.org/10.1007/s11069-019-03834-7

65. Carpinteri, A.; Niccolini, G.; Lacidogna, G. Time Series Analysis of Acoustic Emissions in the Asinelli Tower during Local Seismic Activity. Appl Sci 2018. https://doi.org/10.3390/app8071012

66. Kanamori, H.; Anderson, D.L. Theoretical basis of some empirical relationships in seismology. B. Seismol. Soc. Am. 1975, 65, 1073-1095.

67. Hloupis, G.; Stavrakas, I.; Pasiou, E.D.; Triantis, D.; Kourkoulis, S.K. Natural Time Analysis of Acoustic Emissions in Double Edge Notched Tension (DENT) Marble Specimens. Procedia Eng 2015, 109, 248-256.

68. Hloupis, G.; Stavrakas, I.; Vallianatos, F.; Triantis, D. A preliminary study for prefailure indicators in acoustic emissions using wavelets and natural time analysis. Proc. Inst. Mech. Eng. L.: J. Mater. Des. Appl. 2016, 230, 780-788. doi:10.1177/1464420715575337 
69. Ai-Jing, L.; Peng-Jian, S.; Hua-Chun, Z. Effects of Exponential Trends on Correlations of Stock Markets. Math. Probl. Eng. 2014. https://doi.org/10.1155/2014/340845

70. Silva, F.E.; Gonçalves, L.L.; Fereira, D.B.B.; Rebello, J.M.A. Characterization of failure mechanism in composite materials through fractal analysis of acoustic emission signals. Chaos soliton fract 2005, 26, 481-494. doi:10.1016/j.chaos.2004.12.042

71. Loukidis, A.; Pasiou, E.D.; Sarlis, N.V.; Triantis, D. Fracture analysis of typical construction materials in natural time. Phys. A: Stat. Mech. Appl. 2020.

72. González, S.L.A.; Morsch, I.B.; Masuero, J.R. Didactic Games in Engineering Teaching - Case: Spaghetti Bridges Design and Building Contest. In Proceedings of the 18th International Congress of Mechanical Engineering (COBEM 2005), Ouro Preto, MG, Brazil, 6-11 November 2005. 